\title{
RATIO GEOMETRY, RIGIDITY AND THE SCENERY PROCESS FOR HYPERBOLIC CANTOR SETS.
}

\author{
Tim Bedford And Albert M. Fisher \\ Delft University of Technology; SUNY at Stony Brook
}

June 26, 1994

\begin{abstract}
Given a $\mathcal{C}^{1+\gamma}$ hyperbolic Cantor set $C$, we study the sequence $C_{n, x}$ of Cantor subsets which nest down toward a point $x$ in $C$. We show that $C_{n, x}$ is asymptotically equal to an ergodic Cantor set valued process. The values of this process, called limit sets, are indexed by a Hölder continuous set-valued function defined on D. Sullivan's dual Cantor set. We show the limit sets are themselves $\mathcal{C}^{k+\gamma}, \mathcal{C}^{\infty}$ or $\mathcal{C}^{\omega}$ hyperbolic Cantor sets, with the highest degree of smoothness which occurs in the $\mathcal{C}^{1+\gamma}$ conjugacy class of $C$. The proof of this leads to the following rigidity theorem: if two $\mathcal{C}^{k+\gamma}, \mathcal{C}^{\infty}$ or $\mathcal{C}^{\omega}$ hyperbolic Cantor sets are $\mathcal{C}^{1}$-conjugate, then the conjugacy (with a different extension) is in fact already $\mathcal{C}^{k+\gamma}, \mathcal{C}^{\infty}$ or $\mathcal{C}^{\omega}$. Within one $\mathcal{C}^{1+\gamma}$ conjugacy class, each smoothness class is a Banach manifold, which is acted on by the semigroup given by rescaling subintervals. Conjugacy classes nest down, and contained in the intersection of them all is a compact set which is the attractor for the semigroup: the collection of limit sets. Convergence is exponentially fast, in the $\mathcal{C}^{1}$ norm.
\end{abstract}

\section{Introduction.}

Consider the sequence $C_{n, x}$ of Cantor subsets which nest down toward a point $x$ in a hyperbolic Cantor set $C \subseteq[0,1]$, and which have been affinely rescaled to have left and right endpoints at 0 and 1 . We wish to describe how the geometry of these sets changes as $n$ increases. If $C$ is a linear set like the middle-third set this is not so difficult to do (we always get just another copy of $C$ !) but, as we shall see, with nonlinearity the behavior of this "scenery process" gets much more interesting.

A different way to describe the small-scale structure of $C$ is by the scaling function, introduced by Feigenbaum for a specific class of examples, and studied by D. Sullivan in the present setting of $\mathcal{C}^{1+\gamma}$ hyperbolic Cantor sets.

A third approach is to define a flow, the continuous dynamics of which reflect the geometrical notion of zooming continuously down toward a point. Ergodicity of the flow implies analogues of the Lebesgue density theorem, proved in [BF 1] for Brownian zero sets and hyperbolic $\mathcal{C}^{1+\gamma}$ Cantor sets; the number one gets (order-two density) is a conformal invariant and provides a measure of the lacunarity of the fractal; compare [Mand]. This scenery flow is constructed for hyperbolic Cantor sets in [BF 2], [BF 3], for hyperbolic Julia sets in [BFU], and for limit sets of geometrically finite Fuchsian and Kleinian groups in [Fi 2]. The associated translation (rather than dilation) scenery flows are studied for hyperbolic $\mathcal{C}^{1+\gamma}$ Cantor sets and the Fuchsian limit sets in [Fi 2] and [Bu-F], where theorems like those of [Fi 1] are proved (an order-two ergodic theorem, and infinite-measure unique ergodicity).

In the present paper we will take a viewpoint close to that of Sullivan in [Su 1], constructing the scenery process in a similar way to the scaling function. It is also possible to work in the other direction; in the later papers [BF 2], [BF 3] we show how to derive the scaling function from the scenery flow, and conversely how to construct the scenery flow from the scaling function. From this point of view the scenery process will be seen as an intermediate object, serving to connect the scenery flow with the scaling function.

Sullivan's main motivation in [Su 1] was to begin to develop a Teichmüller theory for Cantor sets, for use in a new, more "conceptual" proof of the Feigenbaum-Coullet-Tresser conjectures (see [Su 2] and [deMvS]). (Lanford's proof [L] uses (rigorous) computer-assisted estimates). A first step would be to classify 
differentiable structures on the attracting Cantor sets of folding maps. This classification should be in terms of some invariant which would serve as a "modulus" of the structure; the next step would be to put a complex structure on the set of moduli as in classical Teichmüller theory, see [Su 3]. Now for the particular case of the folding map which is the Feigenbaum-Coullet-Tresser renormalization fixed point, the Cantor set not only has a folding dynamics but also a hyperbolic $\mathcal{C}^{1+\alpha}$ dynamics. (This observation is attributed by Rand [Ra] to Misiurewicz). One is thus led to the following separate question, which is the subject of $\S \S 1-3$ of [Su 1]: for general hyperbolic $\mathcal{C}^{1+\alpha}$ Cantor sets, can one classify differentiable structures? Sullivan shows this can be done, with the "modulus" being a bounded Hölder scaling function.

Our own main focus is somewhat different. We want to describe the exact geometry, at small scales, of the Cantor sets, whereas to the differentiable structure, all smoothly equivalent Cantor sets will look the same. However for this purpose also, the scaling function contains precisely the information one needs.

Our main theorems (Theorems 5.4, 7.4 and 7.5) concern respectively the scenery process, the smoothness of limit sets, and $\mathcal{C}^{k+\gamma}$ rigidity. We summarize the totality of the resulting picture. Given one $\mathcal{C}^{1+\gamma}$ hyperbolic Cantor set, consider the collection of all Cantor sets which are $\mathcal{C}^{1+\gamma}$ conjugate to it. Within this collection is a distinguished subcollection, its limit sets. The free semigroup on two generators acts on the conjugacy class (by rescaling subsets of the next level); the limit sets are an attractor for this action, and the scenery process can be described as what one sees when walking out a branch of the tree of the semigroup. Limit sets are exactly the ratio sets (see $\$ 2$ ) built from the associated scaling function. Within the big collection are subcollections with higher degrees of smoothness. The big collection forms an infinite-dimensional Banach manifold, naturally identified with a factor of the $\mathcal{C}^{1+\gamma}$-diffeomorphisms of the interval, after one Cantor set has been chosen as a base point. (The diffeomorphisms are the conjugacies to this set). The subcollections nest down as smoothness increases, and by rigidity these smoothness classes are conjugacy classes as well. Contained in the intersection of them all is the collection of limit sets, with the highest possible smoothness. Choosing one of them as a common base point, these subcollections are naturally identified with the $\mathcal{C}^{k+\gamma}$ diffeomorphisms of the interval. Each is a Banach manifold in its own topology, and is dense in a larger collection with respect to its topology. The free semigroup acts on each manifold. Its points are drawn exponentially fast in the $\mathcal{C}^{1}$ norm toward the common attractor: the collection of limit sets, which form a compact subset of the Banach manifold.

In the course of our paper we give careful proofs of several of Sullivan's theorems ([Su 1] is extremely sketchy). In some cases our different point of view leads us to different arguments from those indicated in [Su 1]. We will describe our approach and results more fully after a further explanation of Sullivan's ideas.

\section{Sullivan's differentiable structures.}

We begin with an ordered topological Cantor set, i.e. a space which is homeomorphic and order isomorphic to the usual middle-third Cantor set. For convenience we use $\sum^{+} \equiv \Pi_{0}^{\infty}\{0,1\}$, together with the product topology, and with the lexicographic order. Charts are defined to be order-preserving homeomorphisms into $\mathbb{R}$; two charts $\zeta, \xi$ are $\mathcal{C}^{k+\gamma}$ compatible if $\zeta \circ \xi^{-1}$ extends, with that degree of smoothness, to a diffeomorphism defined on neighborhoods of the embedded sets. A linear $\mathcal{C}(k, \alpha)$ differentiable structure on $\Sigma^{+}$will be a maximal atlas (a maximal compatible collection of charts). Here, following [Su 1], $\mathcal{C}(k, \alpha)$ denotes all maps which are $\mathcal{C}^{k+\gamma}$ for some $\gamma \in(0,1]$. Therefore, a $\mathcal{C}(k, \alpha)$ linear differentiable structure determines and is determined by a class of Cantor sets embedded in the real line, equivalent by $\mathcal{C}(k, \alpha)$ changes of coordinates.

For simplicity, we are restricting our attention to charts which are order preserving and globally defined. We mention that the word "linear" is being used in two ways: when dynamics is introduced on these sets, it will usually be nonlinear; the differentiable stuctures are called linear because they come from embeddings in the line. (Alternative theories might have charts mapping $\Sigma^{+}$to a product of Cantor sets, or to a subset of some fractal curve!)

Via the homeomorphism from $\Sigma^{+}$, an embedded set $C$ comes equipped with the dynamics of the shift map $\sigma$ on $\Sigma^{+}$. The set also inherits from $\Sigma^{+}$a nested hierarchy of intervals, corresponding to finite words (cylinder sets) in $\Sigma^{+}$. Sullivan uses the shift map to define $\mathcal{C}(1, \alpha)$ hyperbolic Cantor sets (see $\S 1$ below) and the nested intervals to define the ratio geometry of a Cantor set. This assigns to each interval the triple $(l, g, r)$ of length ratios of the left subinterval, middle gap and right subinterval respectively. The hypothesis that an embedded Cantor set $C$ is hyperbolic $\mathcal{C}(1, \alpha)$ is enough to show that a limiting ratio 
geometry, recorded by the scaling function, exists. The ratio geometry is bounded away from 0 and 1 , a condition called bounded geometry; hence the limiting values $(a, b, c)$ are also in the interior of the simplex $\Delta=\{(a, b, c): a+b+c=1\}$. Convergence to the scaling function is taken along inverse branches of $\sigma$, which are indexed by points of an abstract topological Cantor set called the dual Cantor set. Thus the scaling function maps the dual Cantor set to $\Delta$, onto a compact subset of its interior. Convergence is exponentially fast, and the scaling function is Hölder continuous.

Locations in the Cantor set correspond to forward images under $\sigma$, since the digits of $\Sigma^{+}$tell whether the orbit of a point lies in the left or right third of $C$. To study smoothness of a conjugacy or an expanding map, one expects of course to use the locations to estimate difference quotients. However since convergence to the scaling function is taken along inverse branches of $\sigma$, as the scale gets smaller and smaller, the locations jump all over the set.

The first remarkable result from $\S \S 1-3$ of $[\mathrm{Su} 1]$ is that while indeed one cannot compute the derivative of the shift map from the scaling function, nevertheless this function contains complete information about $\mathcal{C}(1, \alpha)$ differentiable structures.

More precisely, one has the following. As we have already mentioned, (1) a hyperbolic $\mathcal{C}(1, \alpha)$ Cantor set has a bounded Hölder scaling function. Next (2) this depends only on the differentiable structure i.e. it is the same for $\mathcal{C}(1, \alpha)$ conjugate Cantor sets. Conversely (3) an embedded Cantor set which has a bounded, Hölder scaling function is in fact $\mathcal{C}(1, \alpha)$ hyperbolic. Finally, (4) in this case the $\mathcal{C}(1, \alpha)$ differentiable structure is determined by the scaling function. In other words two hyperbolic $\mathcal{C}(1, \alpha)$ Cantor sets with the same scaling function are $\mathcal{C}(1, \alpha)$ conjugate. In summary, the bounded Hölder scaling function gives an intrinsic characterization of the differentiable structure, in the sense that no embedding need be specified.

Now furthermore, quoting [Su 1]: " ... if the structure admits a $\mathcal{C}(k, \alpha)$ refinement so that the shift is $\mathcal{C}(k, \alpha)$, this structure is also determined uniquely by the same scaling function ..." ". Stated as a result about representatives instead of the entire equivalence class, this can be interpreted as a rigidity theorem: if two $\mathcal{C}(k, \alpha)$ hyperbolic Cantor sets are conjugate by a map which is $\mathcal{C}(1, \alpha)$, then that map (possibly with a different extension to the gaps) is in fact already $\mathcal{C}(k, \alpha)$.

\section{Summary of results.}

We include in this paper careful statements and proofs in particular of (1), (2), (4) above, and of $\mathcal{C}(k, \alpha)$ rigidity. (We mention that our use of the term "rigidity" is different from that in $\S 5$ of [Su 1]). Since we are interested in the geometry of representatives rather than the equivalence class, all these results are stated in terms of the conjugacy of embedded sets rather than the classification of differentiable structures. As we said above, the reason for this emphasis is that our primary goal is to study the scenery process, and all the sets in the scenery process are the same up to conjugacy.

A small technical difference to [Su 1] is that we use $\mathcal{C}^{k+\gamma}$ rather than $\mathcal{C}(k, \gamma)$ throughout. We do this because it gives sharper statements. Thus e.g. for rigidity we show that $\mathcal{C}^{1}$ conjugacy implies $\mathcal{C}^{k+\gamma}$ conjugacy.

In part because of our change in focus, we give a different proof from that suggested in [Su 1] of (4). Each approach has its own advantages. Sullivan's method, a direct estimate of the derivative by difference quotients using sums of gap lengths, gives a unified way of proving (3) as well as (4). However one also needs to cite an extension lemma, which is not included in [Su 1]. On the other hand our approach gives a unified treatment of $\mathcal{C}^{1}$ conjugacy and rigidity, and avoids calling on the separate extension lemma. We do not prove (3) here, but will give a full proof (along the lines of [Su 1]) elsewhere.

Our proof of rigidity is intimately connected to the study of the scenery process. We proceed as follows.

First we use limiting conjugacies to construct a set-valued analogue of the scaling function. This function, $y \mapsto C^{y}$, is defined for $y$ in the dual Cantor set, is Hölder continuous with respect to a metric derived from the corresponding Hausdorff measures and has as its range a compact subset of the collection of all subsets of $[0,1]$ in that measure metric, and also in the Hausdorff metric on sets. The scaling dynamics enters by interpreting the dual Cantor set as the past of the natural extension of the expanding map on $C$; the scenery process $C_{n, x}$ is then asymptotically given by evaluating the shift on any extension $\underline{x}=(y, x)$ of $x \in C$. Since the limit sets were constructed by conjugacies, one can apply a lemma from the appendix of [Su 1] to help determine their degree of smoothness: we show they have the highest degree of smoothness $\left(\mathcal{C}^{k+\gamma}\right.$ for some $k \geq 1, \mathcal{C}^{\infty}$ or $\mathcal{C}^{\omega}$ ) which occurs in the $\mathcal{C}^{1+\gamma}$ conjugacy class of $C$.

Next, the proof of rigidity follows as a corollary. Given two hyperbolic $\mathcal{C}^{k+\gamma}$ Cantor sets, if they are $\mathcal{C}^{1}$ 
conjugate they have the same scaling function. Hence they have the same limit sets, which are ratio Cantor sets constructed from this function. Choosing one of these to act as an intermediary, the composition should also be $\mathcal{C}^{k+\gamma}$. However the maps may be defined differently on the gaps, which would lead back to the extension problem mentioned before. But now one has a simpler solution: a choice is made on the middle third, and the rest of the definition follows automatically from the dynamics. This completes the proof of rigidity. In summary: if two $\mathcal{C}^{k+\gamma}, \mathcal{C}^{\infty}$ or $\mathcal{C}^{\omega}$ hyperbolic Cantor sets are $\mathcal{C}^{1}$ conjugate, then this conjugacy (with a different extension) is already $\mathcal{C}^{k+\gamma}, \mathcal{C}^{\infty}$ or $\mathcal{C}^{\omega}$ respectively.

This leads, then, to the overall picture which is summarized at the end of the first part of the Introduction.

Now one knows, from the rigidity theorem, that the maximum degree of smoothness occuring in the $\mathcal{C}^{1+\gamma}$ conjugacy class should be encoded somehow in the scaling function. Work of Tangerman and Przytycki gives one way of recovering that information [T-P]. A. Pinto and D. Rand ([P-R 2], $\S 5$ and personal communication) and Dennis Sullivan (personal communication) have suggested other approaches, in a related situation. It would be nice to understand in a unified way these different points of view.

The rigidity theorem is also stated by Tangerman and Przytycki; it is proved as a corollary of their main result. Their approach is quite different from ours and in particular does make use of Whitney's Extension Theorem. (We became aware of their preprint after the first version of this paper - an IHES preprint, July 1992 - was completed).

Rand introduces the notion of a Markov family to help study the relationship between scaling functions and smooth conjugacy in situations where one has a sequence of expanding maps, rather than a single map. The examples studied in [Ra], [P], and [P-R 1,2] include certain circle diffeomorphisms and folding maps. See $[\mathrm{AF}]$ for some related developments.

Interesting work on the small-scale geometry of certain fractal sets, in quite different settings, has been done by Hillel Furstenberg and Tan Lei. Tan Lei in $[\mathrm{T}]$ proves the beautiful theorem that certain nonhyperbolic Julia sets, corresponding to Misiurewicz points in the boundary of the Mandelbrot set $\partial M$, are asymptotically the same as $\partial M$ at that point. These points form a countable dense subset of $\partial M$, yet the general case is still far from completely understood. This type of asymptotic limit, as well as what we have called here limit sets, provide examples of Furstenberg's general notion of the microsets of a subset of Euclidean space (lectures and personal communication). These are by definition all the limiting sets given by rescaling nested subsets by a sequence of affine expansions. Furstenberg applies this in a continuation of the analysis begun in $[\mathrm{Fu}]$ for determining the Hausdorff dimension of certain sets: intersections of generic translates of linear Cantor sets, and intersections of linear Cantor sets in the plane with foliations of straight lines at a generic slope. His study of these matters is related to the "times 2 times 3" circle of problems in Ergodic Theory. An interesting and important area of research is to develop similar results in a nonlinear setting, e.g. for general smooth foliations or for nonlinear Cantor sets.

Acknowledgements. We wish to thank our colleagues and friends for conversations, encouragement and inspiration regarding this and related projects. We give special thanks to M. Urbanski, B. Mandelbrot, S. Kakutani, and D. Sullivan. The second-named author would also like to thank the Dynamical Systems seminar at Memphis State University for their encouragement to give a series of lectures on these topics (Spring 1990), and Yale University, MSRI, IHES, CUNY, the CNRS, Université Paris-Nord, and SUNY at Stony Brook for their support while the paper was being written.

\section{$\S 1$ Two ways of building Cantor sets.}

§1.1 Hyperbolic Cantor sets, Hausdorff and Gibbs measures. We start with the usual middle-third Cantor set. Let $S$ denote the $2-1$ map on the middle-third set $C$ defined by $x \mapsto 3 x(\bmod 1)$. The Hausdorff dimension of $C$ is $d=\log 2 / \log 3$; writing $H^{d}$ for $d$-dimensional Hausdorff measure and $\mu$ for the restriction $\mu=\left.H^{d}\right|_{C}$, we recall that $\mu$ is a Borel probability measure (total mass $=1$ ) which is invariant under $S$. The

triple $(C, S, \mu)$ is canonically isomorphic to the one-sided Bernoulli left shift $\sigma$ on $\Sigma^{+} \equiv \prod_{0}^{\infty}\{0,1\}$, with infinite $\left(\frac{1}{2}, \frac{1}{2}\right)$ coin-tossing measure; the correspondence is given by $\pi:\left(x_{0} x_{1} \ldots\right) \mapsto x$ where $x \in C$ has ternary expansion

$$
x=\sum_{i=1}^{\infty} 2 x_{i} 3^{-i} .
$$


A hyperbolic $\mathcal{C}^{1+\gamma}$ Cantor set $C$ by definition also has an expanding dynamics $S: C \longrightarrow C$, but now instead of having straight lines as for $3 x(\bmod 1)$, the graph of $S$ may be nonlinear:

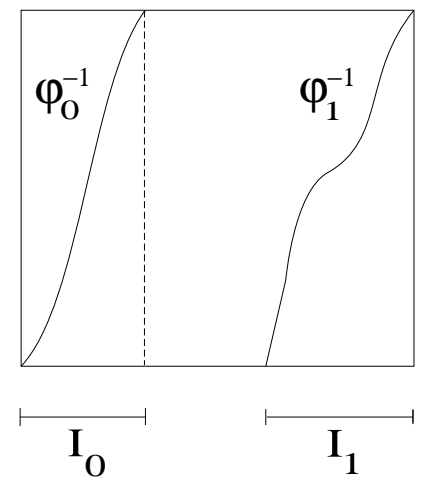

[Figure 1]

To construct such a set, one defines $C$ as a limit from two contraction mappings $\varphi_{0}, \varphi_{1}: I \longrightarrow I$. We consider first the case where these maps are orientation-preserving, and are strict contractions in the sense that the derivatives satisfy $0<\alpha<D \varphi_{i}<\beta<1$. We also require that

$$
0=\varphi_{0}(0)<\varphi_{0}(1)<\varphi_{1}(0)<\varphi_{1}(1)=1 .
$$

This implies that the intervals $I_{0} \equiv \varphi_{0}(I), I_{1} \equiv \varphi_{1}(I)$ are disjoint. We assume that $\varphi_{0}, \varphi_{1}$ are $\mathcal{C}^{1+\gamma}$ maps for some $\gamma \in(0,1]$. Here $\mathcal{C}^{k+\gamma}$ means the $k^{\text {th }}$ derivative $D^{k} \varphi_{i}$ is Hölder continuous with exponent $\gamma$; note that $\mathcal{C}^{1+1}$ means $D \varphi_{i}$ is Lipschitz, so $\mathcal{C}^{2}$ implies $\mathcal{C}^{1+1}$ (by compactness) but not conversely. (Exponent $\gamma>1$ is excluded because in that case, since the domain $I$ is connected, $\varphi_{i}$ is identically constant hence immediately of order $\mathcal{C}^{\infty}$ - while the whole purpose of Hölder conditions is to have intermediate grades of smoothness). Our convention for Hölder continuity will be: if we are given that $f$ satisfies $|f(x)-f(y)| \leq c_{0}|x-y|^{\gamma}$, then we say $f$ is Hölder continuous with Hölder constant $c_{0}$ and Hölder exponent $\gamma$. We remark that Sullivan in [Su 1] works instead with $\mathcal{C}(k, \alpha)$ maps; these are defined to be the collection of $\mathcal{C}^{k+\gamma}$ maps for all $\gamma \in(0,1]$.

We define $S: I_{0} \cup I_{1} \rightarrow I$ to be the map with inverse branches $\varphi_{0}, \varphi_{1}$. Note that since $D \varphi_{i}$ are bounded away from 0 and $\infty$, it follows that $S$ is $\mathcal{C}^{1+\gamma}$ with same Hölder exponent, but with different Hölder constant.

Inductively, form

$$
I_{x_{0} \ldots x_{n}}=\varphi_{x_{0}}\left(\varphi_{x_{1}} \ldots\left(\varphi_{x_{n}}(I)\right)\right)
$$

where $x_{k} \in\{0,1\} ; \cup I_{x_{0} \ldots x_{n}}$ (union over all choices, with $n$ fixed) is the $n^{\text {th }}$ level approximation to the Cantor set, $C$, defined as

$$
C=\bigcap_{n=0}^{\infty} \bigcup I_{x_{0} \ldots x_{n}}
$$

The restriction of the map $S$ to $C$ maps $C$ to itself and is (just as for the middle-third set) conjugate to the Bernoulli shift $\left(\Sigma^{+}, \sigma\right)$, via the map $\pi:\left(x_{0} x_{1} \ldots\right) \mapsto x$, where $x$ is the unique element of $\cap_{n=0}^{\infty} I_{x_{0} \ldots x_{n}}$.

A set $C$ together with map $S: I_{0} \cup I_{1} \rightarrow I$, defined in this way from strict contractions $\varphi_{0}, \varphi_{1}$, will be called a strictly hyperbolic $\mathcal{C}^{1+\gamma}$ Cantor set (with map).

Sometimes we are only interested in the dynamics on $C$ itself. Knowledge of this restricted map $\left.S\right|_{C}$ is equivalent to knowing how $C$ is coded by $\Sigma^{+}$. We will refer to a set together with this labeling as a marked Cantor set. When we forget about this coding, $C$ will be referred to as the underlying Cantor set of $(C, S)$.

More generally, a hyperbolic $\mathcal{C}^{1+\gamma}$ Cantor set is defined as follows. Again we assume that $\varphi_{0}, \varphi_{1}: I \rightarrow I$ are order-preserving $\mathcal{C}^{1+\gamma}$ diffeomorphisms such that $\varphi_{0}(0)=0, \varphi_{1}(1)=1$, and $\varphi_{0}(1)<\varphi_{1}(0)$. We also assume as before that there exists $\alpha$ with $0<\alpha<D \varphi_{i}<1$. However now the upper bound is replaced by one of the two equivalent conditions which follow. We write $\varphi_{x_{0} \ldots x_{n}} \equiv \varphi_{x_{0}} \circ \cdots \circ \varphi_{x_{n}}$. 
Proposition 1.1.1. The following are equivalent:

(i) $\exists \beta<1$ and $N \geq 0$ such that for all $n>N$, for any $x_{0} \ldots x_{n}, D \varphi_{x_{0} \ldots x_{n}}<\beta^{n}$

(ii) $\exists c>0$ and $\tilde{\beta}<1$ such that for all $n$, for any $x_{0} \ldots x_{n}, D \varphi_{x_{0} \ldots x_{n}}<c \tilde{\beta}^{n}$.

Proof. To pass from (ii) to (i), any $\beta$ will work which satisfies $\tilde{\beta}<\beta<1$. For the converse, take $c=$ $\max \left\{\left(D \varphi_{x_{0} \ldots x_{N}}\right) \cdot \beta^{-N}\right\}$ and $\tilde{\beta}=\beta$.

Two $\mathcal{C}^{1+\gamma}$ hyperbolic Cantor sets $C, \widetilde{C}$ with maps $S, \widetilde{S}$ will be said to be continuously conjugate, or $\mathcal{C}^{0}$-conjugate, if there is an order-preserving homeomorphism $\Phi: I \rightarrow I$ such that for all $x \in I_{0} \cup I_{1}$, $\widetilde{S} \circ \Phi(x)=\Phi \circ S(x)$. We say $C, \widetilde{C}$ are $\mathcal{C}^{k+\gamma}, \mathcal{C}^{\infty}, \mathcal{C}^{\omega}$ conjugate if $\Phi$ and its inverse have that degree of smoothness. Note that from the definition, for each $n, \Phi\left(I_{x_{0} \ldots x_{n}}\right)=\widetilde{I}_{x_{0} \ldots x_{n}}$. Therefore the conjugacy induces the identity map on the corresponding shift spaces.

When $\Phi$ is defined (as above) on all of $I$ we will also call it a full conjugacy. A restricted conjugacy is a conjugacy between the Cantor sets which can be extended to a full conjugacy.

Lemma 1.1.2. Let $(C, S)$ be a hyperbolic Cantor set, and assume $\Phi: I \rightarrow I$ is a $\mathcal{C}^{1+\gamma}$ diffeomorphism. Define sets $\widetilde{C} \equiv \Phi(C), \widetilde{I}_{i} \equiv \Phi\left(I_{i}\right)$ for $i=0,1$, and define the map $\widetilde{S}: \widetilde{I}_{0} \cup \widetilde{I}_{1} \rightarrow I$ by $\widetilde{S} \equiv \Phi \circ S \circ \Phi^{-1}$. Then $(\widetilde{C}, \widetilde{S})$ is also a hyperbolic $\mathcal{C}^{1+\gamma}$ Cantor set.

Proof. This is immediate. Note that using condition (i), $\tilde{\beta}$ stays the same but the constant $c$ may change. Similarly, using (ii), $N$ may change while $\beta$ remains the same.

Thus in particular the $\mathcal{C}^{1+\gamma}$ conjugate of a strictly hyperbolic set is still hyperbolic, though strictness may be lost. We remark without proof that a converse holds: by a well-known theorem due in its original form to Mather, any hyperbolic Cantor set is conjugate to a strictly hyperbolic set (without changing the order of differentiability). Therefore if one is studying properties invariant with respect to the equivalence relation given by conjugacy, one might as well begin with the assumption that $c=1$ in (ii); this situation occurs often in the dynamical systems literature. The new metric on $I$ is referred to as an adapted metric for the hyperbolic map $S$. However for our purposes it will be important to use the original metric; otherwise the notion of the scenery process will lose its meaning. This will become clear in $\S 5$.

We recall from the theory of Bowen and Ruelle ([Bo 1,2], [Ru]; see also [Be]) that the dimension $d$ of a hyperbolic $\mathcal{C}^{1+\gamma}$ Cantor set $C$ is strictly between 0 and 1 and that Hausdorff (or conformal) measure $\mu=\left.H^{d}\right|_{C}$ has a unique normalized invariant version $v$, called the Gibbs measure (or Gibbs state). For the middle-third set $\mu=v$; in general they are boundedly equivalent (i.e. the Radon- Nikodym derivative is bounded away from 0 and $\infty$ ); $v$ is defined so as to be a probability measure while $\mu$ may have total mass $\neq 1$. As in $[\mathrm{BF} 1]$, we will need to use both measures.

We remark that all the results in this paper generalize with minor notational changes to the following situation: the maps $\varphi_{i}$ are also allowed to be orientation-reversing; there may be more than two maps, $\varphi_{1}, \ldots, \varphi_{k}$, and the Cantor set is constructed by selecting the maps with respect to some subshift of finite type $\Sigma_{A}$ on $k$ symbols instead of the full two-shift $\Sigma$.

$\S 1.2$ Ratio Cantor sets. As before, $\Sigma^{+}$denotes $\Pi_{0}^{\infty}\{0,1\}$ and now we define: $\Sigma^{-} \equiv \Pi_{-\infty}^{-1}\{0,1\}, \Sigma \equiv$ $\Pi_{-\infty}^{\infty}\{0,1\}$. We will write $y=\left(\ldots y_{-2} y_{-1}\right)$ for $y \in \Sigma^{-}, x=\left(x_{0} x_{1} \ldots\right) \in \Sigma^{+}$and $\underline{x}=(y, x)=\left(\ldots x_{-2} x_{-1} . x_{0} x_{1} \ldots\right)=$ $\left(\ldots y_{-2} y_{-1} \cdot x_{0} x_{1} \ldots\right)$ for a point in $\Sigma$. We will let $\sigma$ denote both the (full) left shift on $\Sigma$ and the left shift (with truncation) on $\Sigma^{+} . \Sigma^{+}$is known as the future of $\Sigma$, and $\Sigma^{-}$as its past.

Write $\Delta$ for the unit simplex in $\mathbb{R}^{3}$ and int $\Delta$ for its interior. Let $R$ be a continuous function from $\Sigma^{-}$to int $\Delta$, and write the components $R=\left(R_{l}, R_{g}, R_{r}\right)$. These letters will stand for left, gap and right respectively; by definition they add to 1 and each is strictly positive. For $\underline{x}=(y, x) \in \Sigma$ we will also think of $R$ as a function on $\Sigma$, by defining $R(\underline{x}) \equiv R(y)$.

Given the function $R$, we will define for each $y \in \Sigma^{-}$the ratio Cantor set $C^{y} \subseteq[0,1]$ so as to satisfy the following: at each stage, subintervals will have length ratios $R\left(\sigma^{n}(\underline{x})\right.$. Thus, we first define $I_{0}^{y}=\left[0, R_{l}(y)\right]$, $I_{1}^{y}=\left[1-R_{r}(y), 1\right]$. The left interval $I_{0}^{y}$ has subintervals $I_{00}^{y}, I_{01}^{y}$ which are defined to have lengths in the ratios

$$
\frac{\left|I_{00}^{y}\right|}{\left|I_{0}^{y}\right|}=R_{l}\left(\ldots y_{-2} y_{-1} 0 .\right), \frac{\left|I_{01}^{y}\right|}{\left|I_{0}^{y}\right|}=R_{r}\left(\ldots y_{-2} y_{-1} 1 .\right)
$$


and left and right endpoints the same as those of $I_{0}^{y}$, respectively. Inductively, for $x \in \Sigma^{+}$and $\underline{x}=(y, x)$, $I_{x_{0} \ldots x_{n+1}}^{y}$ is a subinterval of $I_{x_{0} \ldots x_{n}}^{y}$ with length ratio

$$
\frac{\left|I_{x_{0} \ldots x_{n+1}}^{y}\right|}{\left|I_{x_{0} \ldots x_{n}}^{y}\right|}=R_{*}\left(\sigma^{n} \underline{x}\right) .
$$

Here $*=l$, i.e. this is the left subinterval, if $x_{n+1}=0$, and $*=r$, i.e. the right subinterval, if $x_{n+1}=1$.

Note that the fact that $R$ depends only on the past coordinates $y \in \Sigma^{-}$of $\underline{x}$ is what makes this well-defined, since therefore the ratio is the same for each other point in that subinterval.

Now finally we form the set $C^{y}$ as before, defining

$$
C^{y}=\bigcap_{n=0}^{\infty} \bigcup I_{x_{0} \ldots x_{n}}^{y}
$$

The simplest example is again the middle-third set: taking $R(y)=\left(\frac{1}{3}, \frac{1}{3}, \frac{1}{3}\right)$ for all $y \in \Sigma^{-}, C^{y}$ is the middle-third set $C$ for each $y$.

Since $R$ by assumption is a continous map from a compact set into the interior of $\Delta$, hence strictly into the interior, these intervals $I_{x_{0} x_{1} \ldots}$ nest down to a single point in $[0,1]$. Hence each $C^{y}$ inherits from $\Sigma^{+}$the dynamics of the shift map $\sigma$. (We will also write $\sigma$ for this map on $C^{y}$ ).

For $R$ assumed to be Hölder continuous with some exponent $\alpha>0$ (which will be the case in the present paper), it turns out that each ratio Cantor set $C^{y}$ is also a hyperbolic $\mathcal{C}^{1+\gamma}$ Cantor set. (What needs to be shown is that $\sigma$ is $\mathcal{C}^{1+\gamma}$ on $C^{y}$ for some $\gamma$ with $0<\gamma<1$, which follows by a bounded distortion argument, and that it can be extended to a map $S$ on $I_{0}^{y} \cup I_{1}^{y}$ without losing any smoothness and which is hyperbolic. This can be proved from a lemma of Sullivan, (3) in the Introduction above. For a full proof see [BF 3]). Therefore it also has a Gibbs measure equivalent to Hausdorff measure. The dimension of $C^{y}$ and the Gibbs state, viewed as a measure on $\Sigma^{+}$, are the same for each $y \in \Sigma^{-}$for the following reason: for $y, w \in \Sigma^{-}, C^{y}$ and $C^{w}$ have the same scaling function and hence are $\mathcal{C}^{1+\gamma}$ conjugate. (See $\S 7$ ).

\section{$\S 2$ Statement of the problem; Bounded distortion.}

Let $\mathrm{C}$ be a hyperbolic Cantor set. We wish to describe the geometry of the sequence of nested Cantor sets one sees along the way when zooming down toward a point.

Notation will be as follows: for $x \in C$ with $x=\pi\left(x_{0} x_{1} \ldots\right)$, let $C_{n, x}$ be the set $C \cap I_{x_{0} \ldots x_{n}}$ affinely rescaled to the unit interval, so as to have endpoints at 0 and 1.

Write $\mu_{n, x}$ for the corresponding Hausdorff measure, the restriction of $H^{d}$ to the set $C_{n, x}$. Thus we want to see how the sequence of sets, and of the corresponding measures, varies as $n \longrightarrow \infty$.

The first obstacle we encounter is that you don't get from the interval $I_{x_{0} \ldots x_{n}}$ to its subinterval $I_{x_{0} \ldots x_{n+1}}$ by one application of $\varphi_{0}$ (or $\left.\varphi_{1}\right)$. Instead you have

$$
I_{x_{0} \ldots x_{n+1}}=\varphi_{x_{0}} \ldots \varphi_{x_{n+1}}(I)=\varphi_{x_{0}} \ldots \varphi_{x_{n+1}}\left(\varphi_{x_{n}}^{-1} \ldots \varphi_{x_{0}}^{-1}\left(I_{x_{0} \ldots x_{n}}\right)\right),
$$

and since the maps don't commute, you have to go all the way back up and down the tree again, with more nonlinearity introduced each time. To control this nonlinearity we will use the well-known Bounded Distortion Property, in the following variation. For a proof see [Sh-Su], [Mañé] or Lemma 6.4 below. We learned this version of bounded distortion from M. Urbanski.

Theorem 2.1 (Classical). With $S$ as above, $\exists K>0$ such that for all $n$, for any $\delta>0$, if $J$ is an interval such that $\left.S^{m}\right|_{J}$ is 1-1 and the image $S^{m}(J)$ has diameter less that $\delta$, then for all $x, y \in J$,

$$
e^{-K \delta^{\gamma}}<\left|\frac{D S^{m} x}{D S^{m} y}\right|<e^{K \delta^{\gamma}} .
$$

We mention that one sees from the proof that if $c$ is the Hölder constant for $\log |D S|$, then the constant $K$ is given by $K=c \beta^{\gamma} /\left(1-\beta^{\gamma}\right)$.

As a consequence of this theorem, since $0<\alpha<D \varphi_{i}<\beta<1$ implies that $\alpha^{n}<\left|I_{w_{o} \ldots w_{n}}\right|<\beta^{n}$ for any $w$, we have: 
Corollary 2.2. For any $m, n \geq 0$ and any $w \in \prod_{o}^{\infty}\{0,1\}$ one has for all $x, y \in I_{w_{0} \ldots w_{n+m}}$,

$$
e^{-K \beta^{n \gamma}}<\frac{\left|D S^{m}(x)\right|}{\left|D S^{m}(y)\right|}<e^{K \beta^{n \gamma}}
$$

The set $C_{k, x}$ belongs to the collection of $2^{k}$ Cantor sets at level $k$ in the tree (rescaled). We want to understand the geometry of the sets in this collection.

A first approximation is the original set itself (at level 0). But by bounded distortion, for $n$ large the $2^{n}$ sets at level $n$ provide much better models for the $2^{m+n}$ sets at level $k=m+n$; moreover (and this is the strength of bounded distortion), this is true for all $m$ simultaneously. The reason is that since by definition

$$
S^{m}\left(I_{y_{0} \ldots y_{m} x_{0} \ldots x_{n}}\right)=I_{x_{0} \ldots x_{n}}
$$

and since $I_{x_{0} \ldots x_{n}}$ has small diameter, by the Corollary the derivative of $S^{m}$ is close to constant - hence $S^{m}$ is close to linear.

In summary, consider all the Cantor subsets which have this same image under $S^{m}$ to be grouped in one equivalence class. The $2^{k}$ sets at level $k$ are split into $2^{n}$ equivalence classes, each with $2^{m}$ members which all have approximately the same geometry, (but whose locations are scattered throughout the space!). As we scale down toward a point $x$, we are seeing sets given by these approximations.

Note that the equivalence class of a given interval at level $k$ depends on the immediately previous $n$ branches, rather than on its initial branching structure. We will see in the next section how Sullivan uses this observation to study the asymptotics, associating to the Cantor set a function $R$ like that used to define the ratio Cantor sets in the previous section. Then, in $\S 5$, we will show that the sequence of sets one sees in $C$ is asymptotically the same as that for the ratio Cantor sets $C^{y}$.

And now for the set $C^{y}$, the nested sequence of subsets has an exact description. Each subset is itself a ratio Cantor set. Moreover the sequence changes in the following way. Writing as above $C_{n, x}^{y}$ for the set $C^{y} \cap I_{x_{0} \ldots x_{n}}^{y}$ affinely rescaled to $[0,1]$, one has immediately from the definitions that $C_{n, x}^{y}=C^{\sigma^{n}(\underline{x})}$ for all $n \geq 0$. With the Gibbs measure on the full shift, this gives a stationary, set-valued process - which in forward time describes exactly what one sees as one zooms down toward Hausdorff-almost every point in the ratio Cantor set $C^{y}$.

$\S 3$ Sullivan's Scaling Function. Now we return to the study of a hyperbolic Cantor set $C$. Instead of treating the structure of the entire set $I_{x_{0} \ldots x_{n}} \cap C$, which is what we have been emphasising so far, Sullivan focuses on the information contained in the first step of its construction, given by the relative lengths of the subintervals of $I_{x_{0} \ldots x_{n}}$. These subintervals are the left third $I_{x_{0} \ldots x_{n} 0}$, right third $I_{x_{0} \ldots x_{n} 1}$ and middle gap written $G_{x_{0} \ldots x_{n}}$. We normalize the lengths of these three intervals, defining for $x \in C$ and $n \geq 0$, where $x=\pi\left(x_{0} x_{1} \ldots\right)$,

$$
R_{n, x}=\left(\left|I_{x_{0} \ldots x_{n} 0}\right|,\left|G_{x_{0} \ldots x_{n}}\right|,\left|I_{x_{0} \ldots x_{n} 1}\right|\right) /\left|I_{x_{0} \ldots x_{n}}\right| .
$$

This is called by Sullivan the ratio geometry function of $C$; it maps $\mathbb{N} \times C$ to the interior of the unit simplex $\Delta \subseteq \mathbb{R}^{3}$ and determines $C$ uniquely (one simply constructs $C$ to have these ratios).

Next we write, for $y=\left(\ldots y_{-2} y_{-1}\right)$ in $\widetilde{C} \equiv \prod_{-\infty}^{-1}\{0,1\}$,

$$
R_{n}(y)=\left(\left|I_{y_{-n} \ldots y_{-1} 0}\right|,\left|G_{y_{-n} \ldots y_{-1}}\right|,\left|I_{y_{-n} \ldots y_{-1} 1}\right|\right) /\left|I_{y_{-n} \ldots y_{-1}}\right|
$$

Following Sullivan, it is nice to think of $\widetilde{C}$ as a distinct Cantor set, dual to $C$ (and called the dual Cantor set). Later for the dynamical interpretation we will instead view $\widetilde{C}$ as $\Sigma^{-}$, that is, as the past coordinates of the full shift $\Sigma \equiv \prod_{-\infty}^{\infty}\{0,1\}$. We will use whichever symbol $\left(\widetilde{C}\right.$ or $\left.\Sigma^{-}\right)$is more appropriate in the context.

As in [Bo 1], for any $\beta \in(0,1)$, the $\beta$-metric on $\Sigma^{-}$(which defines what is meant below by Hölder continuity) is taken to be: $d_{\beta}(y, w)=\beta^{n}$ where $n$ is the greatest positive integer such that $y_{-n} \ldots y_{-1}=$ $w_{-n} \ldots w_{-1}$. When $\Sigma^{-}$is thought of as $\widetilde{C}$, i.e. as dual to a specific hyperbolic Cantor set $C$, we choose $\beta$ to 
be (as before) the upper bound on $D \varphi_{i}$. We mention that if $\beta$ is replaced by some other number $\tilde{\beta} \in(0,1)$, then the metrics are related by

$$
d_{\tilde{\beta}}=\left(d_{\beta}\right)^{\log \tilde{\beta} / \log \beta},
$$

and the Hölder exponent $\gamma$ for $R$ in the statement of the next theorem would change to $\gamma \cdot(\log \tilde{\beta} / \log \beta)$.

Theorem 3.1 (Sullivan). Let $C$ be a hyperbolic $\mathcal{C}^{1+\gamma}$ Cantor set. For every $y$ in the dual Cantor set $\widetilde{C}$,

$$
R(y) \equiv \lim _{n \rightarrow \infty} R_{n}(y)
$$

exists. The convergence is of order $O\left(\beta^{n \gamma}\right)$, uniformly in $y$, and the function $R$ is Hölder continuous with exponent $\gamma$, in the $\beta$-metric. $R$ takes values strictly in the interior of $\Delta$.

Definition. $R$ is called the scaling function of $C$.

Proof. We will first show that for each $y, R_{n}(y) n=1,2, \ldots$ is a Cauchy sequence. Since

$$
S^{m}\left(I_{y_{-(n+m)} \ldots y_{-1}}\right)=I_{y_{-n} \ldots y_{-1}}
$$

and similarly for the subintervals, applying the Mean Value Theorem and Bounded Distortion Property (Corollary 2.2) we have for all $m \geq 0$

$$
R_{n}(y)=R_{n+m}(y) e^{ \pm K \beta^{n \gamma}}
$$

Therefore $R_{n}(y)$ is Cauchy sequence (i.e. each of its three coordinates is) hence it converges; call the limit $R(y)$. Next, if $y, w \in \prod_{-\infty}^{-1}\{0,1\}$ agree on the coordinates $-n, \ldots,-1$ then since $R_{n}(y)=R(y) e^{ \pm K \beta^{n \gamma}}$ and $R_{n}(y)=R_{n}(w)$, we have

$$
R(y)=R(w) e^{ \pm 2 K \beta^{n \gamma}} .
$$

Writing $\|\cdot\|$ for sup norm in $\mathbb{R}^{3}$, this implies that, with the log taken by components,

$$
\|\log R(y)-\log R(w)\| \leq 2 K\left(d_{\beta}(y, w)\right)^{\gamma},
$$

i.e. $\log R$ is Hölder continuous with exponent $\gamma$; therefore so is $R$.

Thus (since the normalized lengths add to one), $R$ maps $\widetilde{C}$ onto a compact subset of the interior of the unit simplex in $\mathbb{R}^{3}$.

$\S 4$ Dynamical versions of Sullivan's theorem. In this and the next section we return to the original motivating question: what does the sequence of sets $C_{n, x}$ look like? This is exactly what one sees for the $n^{\text {th }}$ level Cantor set, as one zooms down toward $x$.

First we state Theorem 3.1 in a dynamical form. Here it will be crucial to think of the dual Cantor set $\widetilde{C}$ as the past $\Sigma^{-}$of $\Sigma$. We extend the function $R$ to $\Sigma$ by defining: $R(\underline{x})=R(y)$ for $\underline{x}=(y, x)$. This function depends only on the past coordinates y of $\underline{x}$.

Corollary 4.1. For each $x \in C$, for any choice of $\underline{w} \in \Sigma$ such that $\exists k \geq 0$ with $w_{k}, w_{k+1} \cdots=x_{k}, x_{k+1} \ldots$ (where $\left.x=\pi\left(x_{0} x_{1} \ldots\right)\right)$, then

$$
\left\|R_{n, x}-R\left(\sigma^{n} \underline{w}\right)\right\| \longrightarrow 0 \text { as } n \longrightarrow \infty .
$$

The proof is immediate from the definitions, and in fact if the Hölder constant for $R$ is $c>0$ so that

$$
\|R(\underline{x})-R(\underline{z})\| \leq c d_{\beta}(\underline{x}, \underline{z})
$$

one has

$$
\left\|R_{n, x}-R\left(\sigma^{n}(\underline{w})\right)\right\| \leq c \beta^{n-k}
$$


here the $\beta$-metric has been extended to $\Sigma$ in the natural way, with points $\underline{x}, \underline{w}$ having to agree on coordinates from $-n$ to $n$.

We note that equivalently, if $x$ and $w$ are in the same unstable set in $(C, S)$ then the sequence $R_{n, x}$ (with any past) is in the stable set (in the shift on sequence space) of the sequence given by $R$ sampled along the shift orbit of $\underline{x}$, with an exponential rate of convergence.

We recall that a stochastic process is simply a (one- or two-) sided sequence of measurable functions $f_{i}$ (known as random variables) defined on some probability space $(\Omega, \nu)$. The process is stationary if a timeshift doesn't alter the probability of an event. Equivalently, the space of paths $\left\{\left(\ldots, f_{i}(\omega), \ldots\right)\right\}$, acted on by the shift tranformation and given the pushed-forward measure, is a measure-preserving transformation of a probability space. Conversely, a measure-preserving transformation determines many stochastic processes: choose a measurable function and evaluate it along orbits. Thus for example $R\left(\sigma^{n} \underline{w}\right)$ is a ( $\Delta$-valued) stochastic process. In the next section we will encounter set- and measure-valued versions of this.

Next, recall the definition of a generic point $x$ for an ergodic measure-preserving transformation $T$ on a compact metric space $X$ with probability measure $m$. For each continuous $f: X \rightarrow \mathbb{R}, x$ satisfies:

$$
\lim _{N \rightarrow \infty} \frac{1}{N} \sum_{k=0}^{N-1} f\left(T^{k} x\right)=\int_{X} f d m .
$$

That is, $x$ samples each continuous function well with respect to time averages. If $X$ is a Polish space (a complete separable metric space) - this will occur in the next section -, then we instead sample the continuous functions with compact support. By the remarks in the previous paragraph, this definition also makes sense for a stationary ergodic stochastic process, if the path space has been given the topology of a Polish space. In the definition of generic point we only take time averages toward $+\infty$, so in the case of a two-sided stochastic process, it will be natural to allow a one-sided sequence as a generic point, as well.

If the measure lives in a compact part of the space (which will always be the case in this paper) then by an ergodic theorem of Kryloff and Bogliouboff (i.e. by the Birkhoff ergodic theorem plus compactness), $m$-almost every $x$ is a generic point.

Now $\mu$ is equivalent to the Gibbs measure $\nu$, which is invariant and has a unique invariant natural extension $\hat{\nu}$ on $(\Sigma, \sigma)$. Hence by Kryloff and Bogliouboff:

Corollary 4.2. For $\mu$ - a.e. $x \in C$, the (one-sided) sequence $R_{1, x}, R_{2, x} \ldots$ is a generic point for the ergodic $\Delta$-valued process $R\left(\sigma^{n}(\underline{w})\right)$ for $n \in \mathbb{Z}$, given by $\underline{w} \in \Sigma$ being distributed like $\hat{\nu}$.

$\S 5$ Conjugacies, and the scenery process. In this section we will construct a set-valued version of the scaling function, and use it to prove analogues of Corollaries 4.1 and 4.2, which will describe how the sequence of sets $C_{n, x}$ approximates the scenery process. We will make use of three different metrics on collections of Cantor sets. One metric, which is derived from the $C^{1}$ norm on the space of conjugacies of Cantor sets, is well suited to proofs and is natural from an abstract point of view. There we will prove properties (convergence at at exponential rate; Hölder dependence) which will then pass over to two geometrically defined metrics: the Hausdorff metric, and a metric derived from the Hausdorff measures.

Three metrics. Our metrics will be defined on several different spaces. Fix a hyperbolic $\mathcal{C}^{1+\gamma}$ Cantor set with map, $(C, S)$. We write $\mathcal{E}^{1+\gamma} \equiv \mathcal{E}^{1+\gamma}(C)$ for the collection of Cantor sets (with maps) which are $\mathcal{C}^{1+\gamma}$-conjugate to $(C, S)$. (From Lemma 1.1.2, these are also hyperbolic $\mathcal{C}^{1+\gamma}$ Cantor sets). We write $\mathcal{E}_{*}^{1+\gamma}$ for the quotient space of $\mathcal{E}^{1+\gamma}$ where $(C, S)$ and $(C, \widetilde{S})$ are identified if $S=\widetilde{S}$ on $C$. This is the collection of marked Cantor sets conjugate to $C$, or equivalently the pairs $\left(C,\left.S\right|_{C}\right)$ with restricted maps. $\mathcal{E}_{* *}^{1+\gamma}$ will denote the collection of underlying Cantor sets. We write Diff ${ }^{1+\gamma}$ for the $\mathcal{C}^{1+\gamma}$ order-preserving diffeomorphisms of $I$. Given choice of the pair $(C, S)$, Diff ${ }^{1+\gamma}$ projects onto $\mathcal{E}^{1+\gamma}(C)$ in a natural way: $f$ is mapped to $\left(C_{f}, S_{f}\right) \equiv\left(f(C), f \circ S \circ f^{-1}\right)$. This is many-to one because there is some freedom given by the gaps; see Proposition 8.3. We note that the projection from $\mathcal{E}^{1+\gamma}$ to $\mathcal{E}_{*}^{1+\gamma}$ is also many-to-one.

The scenery process can be thought of as taking values in $\mathcal{E}_{*}^{1+\gamma}$, the marked Cantor sets, or in the space of underlying sets $\mathcal{E}_{* *}^{1+\gamma}$. We will first prove convergence in the space of conjugacies, Diff ${ }^{1+\gamma}$; this will then imply convergence in the other spaces. 
First we consider two metrics on $\mathcal{E}_{* *}^{1+\gamma}$. We recall the definition of the Hausdorff metric on the collection of closed subsets of the interval $I$ :

$$
d_{H}(A, B)=\inf \{\epsilon: A+(-\epsilon, \epsilon) \supseteq B \text { and } B+(-\epsilon, \epsilon) \supseteq A\} .
$$

This defines a metric on $\mathcal{E}_{* *}^{1+\gamma}$, and a pseudo-metric on the other spaces defined above.

Next, we define the following metric on the set of finite Borel measures on $[0,1]$, denoted $\mathcal{M}$. Enumerating binary intervals $E_{1}, E_{2}, \ldots, E_{n} \ldots$ of the form $\left[j 2^{-k},(j+1) 2^{-k}\right]$, for $\nu_{1}, \nu_{2}$ in $\mathcal{M}$, set

$$
d\left(\nu_{1}, \nu_{2}\right)=\sum_{n=1}^{\infty}\left|\nu_{1}\left(E_{n}\right)-\nu_{2}\left(E_{n}\right)\right| / 2^{-n}
$$

This metric induces a topology equivalent to the weak topology on $\mathcal{M}$, in the language of probability theory; in analysis terminology this is the weak-* topology on $\mathcal{M}$, the dual of the space of continuous functions.

On $\mathcal{E}_{* *}^{1+\gamma}$ we define the measure metric $d_{M}$ from this, setting:

$$
d_{M}(C, D)=d\left(\left.H^{d}\right|_{C},\left.H^{d}\right|_{D}\right)
$$

where $H^{d}$ is $d$-dimensional Hausdorff measure. On the other spaces, this again defines a pseudo-metric.

Next, recall that the $\mathcal{C}^{1}$-norm of $f: I \rightarrow \mathbb{R}$ is:

$$
\|f\|_{\mathcal{C} 1}=\|f\|_{\infty}+\|D f\|_{\infty} .
$$

We identify Diff ${ }^{1+\gamma}$ with the collection of triples $\left(C_{f}, S_{f}, f\right)$ for $f \in \operatorname{Diff}^{1+\gamma}$, to be written as $\widehat{\mathcal{E}}^{1+\gamma}$. (As we noted above, the map from $\widehat{\mathcal{E}}^{1+\gamma}$ to $\mathcal{E}^{1+\gamma}$ is not one-to-one). The $\mathcal{C}^{1}$-norm on $\operatorname{Diff}^{1+\gamma}$ determines a metric on $\widehat{\mathcal{E}}^{1+\gamma}$ as follows. For $f, g$ in $\operatorname{Diff}^{1+\gamma}$, we write

$$
d_{C}\left(C_{f}, C_{g}\right)=\|f-g\|_{\mathcal{C} 1}
$$

We call this the $\mathcal{C}^{1}$ metric on $\widehat{\mathcal{E}}^{1+\gamma}$.

Note. The metric $d_{C}$ keeps track of the map $S$ on all of its domain $I_{0} \cup I_{1}$, while $d_{H}$ and $d_{M}$ only see the Cantor sets. Thus for the $\mathcal{C}^{1}$ metric, writing $\mathcal{I}$ for the identity map, $(C, S, \mathcal{I})$ and $\left(C, S_{f}, f\right)$ will be a positive distance apart unless in particular $S=S_{f}$ on all of $I_{0} \cup I_{1}$. (Of course also one needs $f=\mathcal{I}$ ).

The definition of $d_{C}$ depends on the initial choice of the set $(C, S)$ (with the identity map); the next proposition shows how the metric varies if we change this "base point" of $\widehat{\mathcal{E}}^{1+\gamma}$. As we will see in $\S 8$, Diff ${ }^{1+\gamma}$ is a Lie group and this is also a statement about bounded invariance of a metric on that group.

Proposition 5.1. Let $D \in \mathcal{E}^{1+\gamma}$, with $D=\Phi(C)$, with $\Phi \in$ Diff ${ }^{1+\gamma}$. We have:

$$
\frac{1}{K} d_{D}<d_{C}<K d_{D}
$$

where $K=2 \max \left\{\|D \Phi\|_{\infty},\left\|D\left(\Phi^{-1}\right)\right\|_{\infty}^{-1}\right\}$.

Proof. We note that from the definition of the $\mathcal{C}^{1}$-norm one has that if $f: I \rightarrow I$ with $f(0)=0$, then $\|f\|_{\mathcal{C} 1} \leq 2\|D f\|_{\infty}$. Therefore if also $g(0)=0$, then $\|g \circ f\|_{\mathcal{C} 1} \leq 2\|g\|_{\mathcal{C} 1}\|f\|_{\mathcal{C} 1}$.

Now for $f, g$ and $\Phi$ as in the statement of the Proposition, we have:

$$
\begin{aligned}
d_{D}\left(C_{f}, C_{g}\right) & =\left\|f \circ \Phi^{-1}-g \circ \Phi^{-1}\right\|_{\mathcal{C} 1}=\left\|(f-g) \circ \Phi^{-1}\right\|_{\mathcal{C} 1} \\
& \leq 2\|f-g\|_{\mathcal{C} 1}\left\|\Phi^{-1}\right\|_{\mathcal{C} 1}=d_{C}\left(C_{f}, C_{g}\right)\left\|\Phi^{-1}\right\|_{\mathcal{C} 1}
\end{aligned}
$$

which gives one of the inequalities. The other is proved in the same way.

Next we will look at how (on $\widehat{\mathcal{E}}^{1+\gamma}$ ) the pseudo-metrics $d_{H}$ and $d_{M}$ compare to the metric $d_{C}$. First we recall how the Hausdorff measure transforms under mappings. 
Definitions. Given a 1-1 differentiable map $\Psi: M \longrightarrow N$ between open subsets of $\mathbb{R}$ and given a Borel measure $\mu$ on $M$ and real number $d>0$ we write: $\left(\Psi^{\star} \mu\right)(E)=\mu\left(\Psi^{-1} E\right)$ and

$$
(\check{\Psi} \mu)(E)=\int_{\Psi^{-1} E}|D \Psi|^{d} d \mu .
$$

Thus $\Psi^{\star} \mu$ is the usual push forward of $\mu$, and $\check{\Psi} \mu$ is the $(\Psi, d)$ - conformal transform of $\mu$.

Hausdorff measure has the conformal transformation property with respect to $\mathcal{C}^{1}$ maps: for $\Psi: \mathbb{R} \rightarrow$ $\mathbb{R}$ a $\mathcal{C}^{1}$ diffeomorphism,

$$
H^{d}=\check{\Psi}\left(H^{d}\right) .
$$

Proposition 5.2. With $d_{H}, d_{M}$ and $d_{C}$ denoting the Hausdorff, measure, and $\mathcal{C}^{1}$ metrics respectively, for all $C_{f}, C_{g} \in \widehat{\mathcal{E}}^{1+\gamma}$,

$$
d_{H}\left(C_{f}, C_{g}\right) \leq d_{C}\left(C_{f}, C_{g}\right)
$$

and for $\Psi(x)=5 x+4 x^{2}$, we have for all $C_{f} \in \mathcal{E}^{r}(C)$,

$$
d_{M}\left(C, C_{f}\right) \leq \Psi\left(d_{C}\left(C, C_{f}\right)\right) .
$$

Proof. For the Hausdorff (pseudo)-metric this is immediate, using the $L^{\infty}$ norm, since

$$
d_{H}\left(C_{f}, C_{g}\right) \leq\|f-g\|_{\infty} \leq d_{C}\left(C_{f}, C_{g}\right)
$$

For the second inequality, writing $\mathcal{I}$ for the identity map on $I$, we have

$$
d_{C}\left(C, C_{f}\right) \equiv\|f-\mathcal{I}\|_{\mathcal{C} 1}
$$

and writing $\mu=\left.H^{d}\right|_{C}, \mu_{f}=\left.H^{d}\right|_{C_{f}}$,

$$
\begin{aligned}
d_{M}\left(C, C_{f}\right) & \equiv \sum_{n}\left|\mu E_{n}-\mu_{f} E_{n}\right| 2^{-n} \\
& =\left.\sum_{n}\left|\int_{f^{-1} E_{n}}\right| D f\right|^{d} \mathrm{~d} \mu-\int_{E_{n}} 1 \mathrm{~d} \mu \mid 2^{-n} \\
& \leq 2\|f-\mathcal{I}\|_{\infty}\|D f\|_{\infty}^{d} \sum_{n} 2^{-n}+\left.\sum_{n} 2^{-n} \int_{f^{-1} E_{n} \cap E_{n}}|| D f\right|^{d}-1 \mid \mathrm{d} \mu
\end{aligned}
$$

(here the first term bounds the contribution, for each interval $E_{n}$, of its two ends not matching up exactly with $f^{-1} E_{n}$; we used the fact that since $f$ is a diffeomorphism of $I,\|D f\|_{\infty} \geq 1$ ).

Next, we note that for all $x>0,\left|x^{d}-1\right| \leq|x-1|$. Hence $\left.|| D f\right|^{d}-1|\leq||D f|-1 \mid$, so the above is

$$
\begin{aligned}
& \leq 2\|f-\mathcal{I}\|_{\infty}\left(1+\||D f|-1\|_{\infty}\right)+\||D f|-1\|_{\infty} \sum_{n}\left|E_{n}\right| 2^{-n} \\
& \leq 4\||D f|-1\|_{\infty}\left(1+\||D f|-1\|_{\infty}\right)+\||D f|-1\|_{\infty} \\
& \leq 4\left(1+\||D f|-1\|_{\infty}+1\right)\left(\||D f|-1\|_{\infty}\right)=5\||D f|-1\|_{\infty}+4\left(\||D f|-1\|_{\infty}\right)^{2} \\
& \leq \Psi\left(d_{C}\left(C, C_{f}\right)\right),
\end{aligned}
$$

as claimed.

We define the (restricted) $\mathcal{C}^{1}$ metric on $\mathcal{E}_{*}^{1+\gamma}$ to be

$$
d_{C *}\left(C_{f}, C_{g}\right)=\|f-g\|_{\mathcal{C} 1, C}
$$

where this indicates that the sup norms are taken over the Cantor set $C$. Obviously $d_{C *} \leq d_{C}$ on $\widehat{\mathcal{E}}^{1+\gamma}$.

The first theorem we are aiming for will state: 
Theorem 5.3. Given a $\mathcal{C}^{1+\gamma}$ hyperbolic Cantor set $(C, S)$, for every $y=\left(\ldots y_{-n} \ldots y_{-1}\right) \in \Sigma^{-}$, the limit

$$
C(y) \equiv \lim _{n \longrightarrow \infty} C_{y_{-n} \ldots y-1}
$$

exists. Convergence is exponentially fast, and $C(y)$ is Hölder continuous with exponent $\gamma$ (for both metrics on $\mathcal{E}_{* *}^{1+\gamma}$, and for the restricted $\mathcal{C}^{1}$ metric on the marked Cantor sets $\left.\mathcal{E}_{*}^{1+\gamma}\right)$. Moreover, $C(y)=C^{y}$, the ratio Cantor set built from the scaling function of $(C, S)$.

Thus $C(y)$ is a set-valued version of the scaling function $R(y)$. To prove Theorem 5.3, we first construct certain conjugacies (Theorem 5.9), proving convergence in the $\mathcal{C}^{1}$ metric. Convergence in the Hausdorff and measure metrics then follows from Proposition 5.2. Before giving this construction, we state several further consequences of Theorem 5.9.

In the same way as for the scaling function $R(y)$, we define $C(\cdot)$ also on the full shift space $\Sigma$, setting $C(\underline{x})=C(y)$ for $\underline{x}=(y, x)$. We will have:

Theorem 5.4. For every $x \in C$, the sequence $C_{n, x} \equiv C_{x_{0} \ldots x_{n}}$ is asymptotic to $C\left(\sigma^{n}(\underline{x})\right)$, with an exponential rate of convergence, for any $\underline{x}$ in $\Sigma$ with the same future coordinates $\left(x_{0}, x_{1}, \ldots\right)$.

We will write $\mathcal{L}_{C}$ for the (compact) subset of $\mathcal{E}_{*}^{1+\gamma}$ (with respect to all three metrics) which is the range of the function $\underline{x} \mapsto C(\underline{x})$. This is the collection of (marked) limit sets. Since the function is continuous and the domain $\Sigma$ is compact, we then have:

Proposition 5.5. The collection of limit sets is compact.

Definition. Given a $\mathcal{C}^{1+\gamma}$ hyperbolic Cantor set $C$, the set-valued scenery process of $C$ is the process $C\left(\sigma^{n}(\underline{x})\right)=C^{\sigma^{n}(\underline{x})}$, with $\underline{x} \in \Sigma$, distributed according to the measure $\hat{\nu}$.

Note that stationarity and ergodicity of this process follow immediately from invariance and ergodicity of the measure $\hat{\nu}$.

The space of paths is a compact subset of the Polish space $\Pi_{-\infty}^{+\infty} \mathcal{E}_{* *}^{1}$ (or $\Pi_{-\infty}^{+\infty} \mathcal{E}_{*}^{1}$ for the marked sets), with the product topology determined by the topologies of any of the three metrics. See the proof of Corollary 5.6 .

We mention why we use probability terminology - the scenery process - for the map $C(\underline{x}) \mapsto C(\sigma \underline{x})$. Note that this dynamics is not in fact given by a map on $\mathcal{L}_{C}$ itself. Indeed at every stage you have two choices - the right- or left-hand subsets from the next level of the ratio Cantor set $C(\underline{x})$, with the choice of left or right depending on whether $x_{0}$ is 0 or 1 . Or, from a different viewpoint, one has the dynamics on $\mathcal{L}_{C}$ of a semigroup action; see the note at the end of the paper.

The next result is like Corollary 4.2.

Corollary 5.6. For $\mu$-a.e. $x \in C$, the sequence of (rescaled) Cantor sets $C_{n, x}$ which nest to $x$ is a generic point for the stationary ergodic set-valued process $C^{\sigma^{n}(\underline{x})}$ determined by $(\Sigma, \sigma, \hat{\nu})$.

In $\S 2$, we defined $\mu_{n, x}$ to be the sequence of Hausdorff measures $\left.H^{d}\right|_{C_{n, x}}$. Convergence of $C_{n, x}$ to $C(y)$ in the measure metric can be rephrased as follows:

Corollary 5.7. For every $y=\left(\ldots y_{-n} \ldots y_{-1}\right) \in \Sigma^{-}$, the limit

$$
M(y) \equiv \lim _{n \longrightarrow \infty} \mu_{y_{-n} \ldots y_{-1}}
$$

exists, and is Hölder continuous with exponent $\gamma$.

The support of the measure $M(y)$ is the set $C(y)$, and $M(y)$ is a measure-valued version of the scaling function.

As we did for the set-valued process, we define $M(\underline{x})=M(y)$. We define the measure-valued scenery process $M(n, \underline{x}) \equiv M\left(\sigma^{n}(\underline{x})\right)$, again with $\underline{x} \in \Sigma$, distributed like $\hat{\nu}$. We have: 
Corollary 5.8. For $\mu$-a.e. $x \in C$, the sequence $\mu_{x_{0} \ldots x_{n}}$ is a generic point for the stationary ergodic measurevalued process $M\left(\sigma^{n}(\underline{x})\right)$ determined by $(\Sigma, \sigma, \hat{\nu})$.

Now we proceed to the proofs. For each interval $I_{w_{0} \ldots w_{n}}$ with $w_{i}=0$ or 1 , we write $A_{w_{0} \ldots w_{n}}$ for the affine map which expands the interval to the unit interval I. We then set for $y \in \widetilde{C}, A_{n}^{y}=A_{y_{-n} \ldots y_{-1}}$. This expands the interval $I_{y_{-n} \ldots y_{-1}}$ to $I$ affinely. Next, define maps $\varphi_{k, n}^{y}: I \rightarrow I$ for $k \geq n$ by:

for $k \geq n$.

$$
\begin{aligned}
& \varphi_{k, k}^{y}=\text { identity and } \\
& \varphi_{k, n}^{y}=\varphi_{y_{k}} \circ \cdots \circ \varphi_{y-(n+1)}
\end{aligned}
$$

We will also write $\varphi_{k}^{y}$ for $\varphi_{k, 0}^{y}$.

For $0 \leq n \leq k$, we define $\Phi_{k, n}^{y}: I \rightarrow I$ by:

$$
\Phi_{k, n}^{y}=A_{k}^{y} \circ \varphi_{k, n}^{y} \circ\left(A_{n}^{y}\right)^{-1} .
$$

It follows that:

$$
\begin{aligned}
& \text { for each } n, \quad \Phi_{n, n}^{y}=\text { identity, } \\
& \text { for all } m \geq k \geq n, \\
& \qquad \begin{array}{l}
\Phi_{m, n}^{y} \\
=\Phi_{m, k}^{y} \circ \Phi_{k, n}^{y} \text { and } \\
\Phi_{n, 0}^{y}=A_{n}^{y} \circ \varphi_{n}^{y} .
\end{array}
\end{aligned}
$$

We will also write $\Phi_{n}^{y}$ for $\Phi_{n, 0}^{y}$.

The sequence $\Phi_{n}^{y}$ is, to use Sullivan's words, "a sequence of ... compositions (of contractions) ... renormalized by post composition with linear maps to obtain mappings between unit intervals..." (Appendix of [Su 1]). As Sullivan states, and as we will prove in the next section, such a sequence is precompact in $\mathcal{C}(k, \alpha)$ if the original hyperbolic Cantor set is $\mathcal{C}(k, \alpha)$.

This gives convergence along some subsequence $\Phi_{n_{k}}^{y}$. However unfortunately, that is not enough for our goal of proving an ergodic theorem for Cantor sets and measures (i.e. Corollaries 5.6, 5.8) - for that purpose we want instead to prove that the sequence itself converges. We do this in the next theorem, using bounded distortion, and then in the next section we return to Sullivan's idea to prove smoothness of the resulting limiting conjugacy.

Theorem 5.9. Let $(C, S)$ be a $\mathcal{C}^{1+\gamma}$-hyperbolic Cantor set. For each $y$ in the dual Cantor set $\widetilde{C}$,

$$
\Phi^{y} \equiv \lim _{n \rightarrow \infty} \Phi_{n}^{y}
$$

exists. This is an order-preserving diffeomorphism from I to I. Convergence is of order $O\left(\beta^{\text {n } \gamma}\right)$ in the $\mathcal{C}^{1}$ norm, uniformly in $y$, and the function $y \mapsto \Phi^{y}$ is Hölder continuous of order $\gamma$, in the $\beta$-metric.

Proof. We will show, using bounded distortion, that for $n$ large and for $k>n$ arbitrary, $\Phi_{k, n}^{y}$ is close to the identity. Then since $\Phi_{k, 0}^{y}=\Phi_{k, n}^{y} \circ \Phi_{n, 0}^{y}$, this will imply convergence.

Now since the maps $A$ are affine they have constant derivative. So for each $a \in I$, for $m=k-n$, we have:

$$
\begin{aligned}
\left(D\left(\Phi_{k, n}^{y}\right)\right)(a) \equiv D\left(A_{k}^{y} \circ\left(S^{m}\right)^{-1} \circ\left(A_{n}^{y}\right)^{-1}\right)(a) & =\frac{D A_{k}^{y}}{D A_{n}^{y} D S^{m}(z)} \text { for } z \equiv\left(A_{n}^{y}\right)^{-1}(a) . \\
& =\frac{D S^{m}\left(z_{0}\right)}{D S^{m}(z)} \text { for some } z_{0} \in I_{y_{-k} \ldots y-1}
\end{aligned}
$$

by the Mean Value Theorem. Therefore by Corollary 2.2

$$
e^{-K \beta^{n \gamma}}<D \Phi_{k, n}^{y}(a)<e^{K \beta^{n \gamma}}
$$


for all $k>n, y \in \widetilde{C}$ and all $a \in I$. This implies the sequence $D \Phi_{n}^{y}, n=0,1,2 \ldots$ is Cauchy, hence converges. Since $\Phi_{n}^{y}(0)=0$ for all $n$, it follows from the Fundamental Theorem of Calculus that the limit $\Phi^{y} \equiv \lim _{n \rightarrow \infty} \Phi_{n}^{y}$ exists, and that $D \Phi^{y}=\lim D \Phi_{n}^{y}$. By Corollary 2.2, $D \Phi^{y}$ is bounded away from 0 and $\infty$ by $e^{ \pm K \beta^{\gamma}}$; in particular, $\Phi^{y}$ is an order-preserving diffeomorphism from $I$ to $I$, as claimed.

We define for each $n$

$$
\Phi_{\infty, n}^{y}=\lim _{k \rightarrow \infty} \Phi_{k, n}^{y}
$$

the limit exists by the above arguments, and this map is $\mathcal{C}^{1}$-close to the identity map $\mathcal{I}: I \rightarrow I$. We have for each $n$ that

$$
\Phi^{y}=\Phi_{\infty, n}^{y} \circ \Phi_{n, 0}^{y},
$$

and that:

(1) $\exists k_{0}>0$ such that

$$
\left\|\Phi_{\infty, n}^{y}-\mathcal{I}\right\|_{\mathcal{C} 1}<k_{0} \beta^{n \gamma} .
$$

The constant $k_{0}$ here only depends on $K$ from Corollary 2.2, which in turn depends on $\beta$, the upper bound for $\left|D \varphi_{i}\right|$. Here is the calculation: we have

$$
\left\|\Phi_{\infty, n}^{y}-\mathcal{I}\right\|_{\mathcal{C} 1} \leq\left\|D \Phi_{\infty, n}^{y}-1\right\|_{\infty}
$$

and we know that

$$
e^{-K} \leq e^{-K \beta^{n \gamma}} \leq D \Phi_{\infty, n}^{y} \leq e^{K \beta^{n \gamma}} \leq e^{K}
$$

for all $n$. Now since for $x$ in the interval $\left[e^{-K}, e^{K}\right]$ one has $\left|e^{x}-1\right|<k_{0} x+1$, where we take $k_{0}=$ $(\exp (\exp (K))-1) /(\exp (K))$, statement $(1)$ holds true.

Now recall from the proof of Proposition 5.1 that for $f, g: I \rightarrow I$ with $f(0)=0$ and $g(0)=0$ then $\|g \circ f\|_{\mathcal{C}_{1}} \leq 2\|g\|_{\mathcal{C}_{1}}\|f\|_{\mathcal{C}_{1}}$. From this, it follows that statement (1) is equivalent to:

(2) $\exists k_{1}>0$ such that

$$
\left\|\Phi_{n}^{y}-\Phi^{y}\right\|_{\mathcal{C}_{1}}<k_{1} \beta^{n \gamma} .
$$

(Here we can take $\left.k_{1}=\left(e^{K}\right) k_{0}\right)$.

Both statements express, in different ways, that $\Phi^{y}$ is close to $\Phi_{n, 0}^{y}$, with exponentially fast convergence; (2) is what we stated in the Theorem. Finally it is now also easy to check Hölder continuity: $\exists k_{2}>0$ such that for all $y, w \in \widetilde{C}$,

$$
\left\|\Phi^{y}-\Phi^{w}\right\|_{\mathcal{C} 1} \leq k_{2}\left(d_{\beta}(y, w)\right)^{\gamma}
$$

Proof of Theorem 5.3. Writing $C_{n}^{y}$ for $\Phi_{n}^{y}(C)=C_{y_{-n} \ldots y_{-1}}$, and $C^{y} \equiv \Phi^{y}(C)$, statement (2) above says exactly:

$$
d_{C}\left(C_{n}^{y}, C^{y}\right)<k_{1} \beta^{n \gamma} .
$$

Hence we have convergence in the $\mathcal{C}^{1}$ metric. By Proposition 5.2 therefore, $d_{H}$ has the same bound. For the measure metric, we have

$$
\begin{aligned}
d_{M}\left(C_{n}^{y}, C^{y}\right) & \leq \Psi\left(d_{C^{y}}\left(C^{y}, C_{n}^{y}\right)\right) \\
& =\Psi\left(\left\|\Phi_{\infty, n}^{y}-\mathcal{I}\right\|_{\mathcal{C} 1}\right) \\
& \leq \Psi\left(k_{0} \beta^{n \gamma}\right)
\end{aligned}
$$

by (1), and this is $\leq k_{3} \beta^{n \gamma}$ where $k_{3}=k_{0}\left(5+4 k_{0}\right)$. Next we show Hölder continuity. Now $d_{C}\left(C^{y}, C^{w}\right)=$ $\left\|\Phi^{y}-\Phi^{w}\right\|_{\mathcal{C} 1}$ so (3) proves Hölder continuity for the $\mathcal{C}^{1}$ and Hausdorff metrics. Then, applying Proposition $5.1, d_{M}\left(C^{y}, C^{w}\right) \leq \Psi\left(d_{C^{y}}\left(C^{y}, C^{w}\right)\right) \leq \Psi\left(d_{C}\left(C^{y}, C^{w}\right)\left\|\Phi^{-1}\right\|_{\mathcal{C} 1}\right) \leq \Psi\left(e^{K} k_{2} \beta^{n \gamma}\right) \leq k_{4} \beta^{n \gamma}$ where $k_{4}=5 a+4 a^{2}$ and $a=e^{K} k_{2}$. Finally, it is clear from the constructions that $C(y)$ has ratio geometry given by $R(y)$, hence $C(y)$ is indeed equal to $C^{y}$.

Proof of Theorem 5.4. From the proof of Theorem 5.3, since the exponential bound is uniform over all sets of level $n$, we have that the $d_{C}$-distance from $C\left(\sigma^{n} x\right)$ to $C_{x_{0} \ldots x_{n}}$ is bounded by $k_{1} \beta^{n \gamma}$. The bounds for $d_{H}$ and $d_{M}$ then follow as above. 
Proof of Corollary 5.6. We give the proof for the process which takes values in the collection of marked sets. Here the space will be $\Pi_{-\infty}^{\infty} \mathcal{E}_{*}^{1}(C)$, with the shift transformation; this is a Polish space (since $\mathcal{E}_{*}^{1}$ is; we use $\mathcal{E}_{*}^{1}$ rather than $\mathcal{E}_{*}^{1+\gamma}$ so as to have a complete space). Therefore we know from our definitions what it will mean for the one-sided sequence $C_{n, x}$ to be a generic point. Now the map $\underline{x} \mapsto\left(\ldots C\left(\sigma^{-1} \underline{x}\right), C(\underline{x}), C(\sigma \underline{x}) \ldots\right)$ from $\Sigma$ has as its image a compact invariant subset of $\Pi_{-\infty}^{\infty} \mathcal{L}_{C} \subseteq \Pi_{-\infty}^{\infty} \mathcal{E}_{*}^{1}(C)$; this image is the space of paths of the scenery process, and is the support of the image of the measure $\hat{\nu}$. The ergodicity of $(\Sigma, \hat{\nu}, \sigma)$ passes over to the scenery process, hence a.e. path $\left(\ldots C\left(\sigma^{-1} \underline{x}\right), C(\underline{x}), C(\sigma \underline{x}) \ldots\right)$ is generic for the shift on path space. Finally, since by Theorem 5.4 we know the sequence $C_{n, x}$ is forward asymptotic to $C\left(\sigma^{n} \underline{x}\right)$, we will compute the same time average for the continuous functions. Thus $C_{n, x}$ is generic, for $\nu$-a.e. $x$ and hence for $\mu$-a.e. $x$.

$\S$ 6. Smoothness of conjugacies. Now we will see how to prove the conjugacies of $\S 5$ in fact have higher smoothness properties.

The basic idea will be to imitate what one knows about analytic maps, for $\mathcal{C}^{k+\gamma}$ or $\mathcal{C}(k, \gamma)$ maps. Thus Lemma 6.2 is a version of Leibnitz' formula, and Lemma 6.3 is one step in showing $\mathcal{C}^{k+\gamma}$ maps are morphisms in a category. This means they can be used to define equivalence relations on sets, and to give the analogue of differentiable structures. One also imitates the Arzela-Ascoli Theorem, in Lemma 6.4; as Sullivan says in the Appendix of [Su 1], and quoted in the previous section, the sequence $\Phi_{n}^{y}$ will be precompact in $\mathcal{C}(k, \alpha)$. (As usual, we do our proofs instead in $\mathcal{C}^{k+\gamma}$ ).

Here is the main theorem we are aiming for.

Theorem 6.1. Let $C$ be a $\mathcal{C}^{k+\gamma}$ hyperbolic Cantor set, and let $\Phi^{y}: I \rightarrow I$ be defined as in $\S 5$. We claim:

(i) if $k=1,2, \ldots$, then $\Phi^{y}$ is $\mathcal{C}^{k+\gamma}$ (with same Hölder exponent, but a different Hölder constant).

(ii) if $k=\infty$ or $\omega$, then $\Phi^{y}$ is $\mathcal{C}^{\infty}$ or $\mathcal{C}^{\omega}$ respectively.

First we need a few lemmas.

Lemma 6.2. For $A \subseteq \mathbb{R}$, if $f: A \rightarrow \mathbb{R}$ and $g: A \rightarrow \mathbb{R}$ are bounded, $\gamma$-Hölder continuous with Hölder constants $c, d$, then:

(i) $f+g$ is $\gamma$-Hölder with constant $c+d$, and

(ii) $f \cdot g$ is $\gamma$-Hölder with constant $c\|g\|_{\infty}+d\|f\|_{\infty}$.

Proof. (i) is immediate. The argument for (ii) comes by imitating the proof of Leibnitz' rule in the Calculus:

$$
\begin{aligned}
|f(x) g(x)-f(y) g(y)| & =|[f(x)-f(y)] g(x)+f(y)[g(x)-g(y)]| \\
& \leq c|x-y|^{\gamma}\|g\|_{\infty}+d|x-y|^{\gamma}\|f\|_{\infty} .
\end{aligned}
$$

Lemma 6.3. Fix $k \geq 1$. For $A, B \subseteq \mathbb{R}$, let $f: A \rightarrow B$ and $g: B \rightarrow \mathbb{R}$ be such that $D^{k}(f)$, $D^{k}(g)$ are bounded and $\gamma$-Hölder. Then $D^{k}(g \circ f)$ is $\gamma$-Hölder.

Proof. This now follows by induction, from the Chain Rule plus Lemma 6.2.

The next lemma is basically the same as the "bounded variation" lemma, Lemma 1.15 from [Bo 1], except it is written in the reverse direction, for the contractions $\varphi_{i}$ instead of the inverse map $S$. For the special case $f_{i}=\varphi_{w_{j}}$ and $h_{i}=\log \left|D \varphi_{w_{j}}\right|$ where $i+j=n$, one gets exactly the Bounded Distortion Property (Theorem 2.1). The formulation given here is from the Appendix of [Su 1]; the key idea for proving our Theorem 6.1, which is also in that Appendix (the sentence immediately preceding the Corollary there), will be how to use this lemma to control higher order derivatives of the composition. One can summarize the idea as follows: do not look at $\log \left|D^{k} \varphi_{x_{i}}\right|$, but instead at $D^{k-1} \log \left|D \varphi_{x_{i}}\right|$. Then we are applying the linear operator $D^{k}$ to a sum, which leads to the proof.

As usual, for notational simplicity, we assume strict hyperbolicity.

Lemma 6.4. Consider a composition of contractions $f_{n} \circ \cdots \circ f_{1}$, with $f_{i}: J_{i} \rightarrow J_{i+1}$ for intervals $J_{i} \subseteq \mathbb{R}$ , and such that $\left|D f_{i}\right|<\beta<1$. For a point $x \in J$, write $x_{1}=x, x_{i+1}=f_{i}\left(x_{i}\right)$. Let $h_{i}: J_{i} \rightarrow \mathbb{R}$ be Hölder 
continuous with the same exponent $\gamma \in(0,1]$ and same constant $c$. Then for $h(x) \equiv h_{1}\left(x_{1}\right)+\cdots+h_{n}\left(x_{n}\right)$, $h$ is also $\gamma$-Hölder continuous, with constant $c_{0}=c \beta^{\gamma} /\left(1-\beta^{\gamma}\right)$ (independent of $n$ ).

Proof. Immediate from the geometric series, since for $x, y \in J_{1}$ we have $\left|x_{i}-y_{i}\right|<\beta^{i}$.

The next little lemma is more subtle than one might at first think. We wish to thank Z. Nitecki and M. Urbanski for discussions which resulted in a first proof, and Y. Kifer for then finding the much simpler argument given here.

Lemma 6.5. Let $f_{n}: I \rightarrow I$ be continuous functions with continuous $k^{\text {th }}$ derivative and assume that there exist functions $f, g$ such that:

(i) $f_{n} \rightarrow f$ and

(ii) $D^{k} f_{n} \rightarrow g$, uniformly as $n \rightarrow \infty$. Then $D^{k} f=g$.

Proof. We define, for each $0 \leq j \leq k$, functions $g_{j}$, and sequences of functions $f_{n, j}$ and $p_{n, j}$ by:

$$
\begin{aligned}
& g_{k}=g \text { and } g_{j-1}(t)=\int_{0}^{t} g_{j} ; \\
& f_{n, k}=D^{k} f_{n} \text { and } f_{n, j-1}(t)=\int_{0}^{t} f_{n, j} ; \\
& p_{n, j}=D^{j} f_{n}-f_{n, j} .
\end{aligned}
$$

Thus

$$
\begin{aligned}
& p_{n, k} \equiv 0 \\
& p_{n, k-1}(t) j=\left(D^{k-1} f_{n}\right)(t)-\left(\left(D^{k-1} f_{n}\right)(t)-\left(D^{k-1} f_{n}\right)(0)\right) \equiv\left(D^{k-1} f_{n}\right)(0), \\
& p_{n, k-2}(t)=D^{k-2} f_{n}(0)+t\left(D^{k-1} f_{n}\right)(0)
\end{aligned}
$$

and similarly, (for each $n$ ) $p_{n, j}$ is for all $j$ a polynomial of degree $k-j-1$, such that $D p_{n, j}=p_{n, j+1}$. Now for each $j, \lim _{n \rightarrow \infty} f_{n, j}=g_{j}$. In particular,

$$
g_{0}=\lim _{n \rightarrow \infty} f_{n, 0}=\lim _{n \rightarrow \infty}\left(f_{n}-p_{n, 0}\right)=f-\lim p_{n, 0} .
$$

Hence $\lim _{n \rightarrow \infty} p_{n, 0}$ converges (uniformly), so to some polynomial $p_{0}$ with degree at most $k-1$, and we have $g_{0}=f-p_{0}$. Therefore,

$$
g=D^{k} g_{0}=D^{k} f-D^{k} p_{0}=D^{k} f
$$

as claimed.

Proof of Theorem 6.1. Since $D^{k} \varphi_{0}$ and $D^{k} \varphi_{1}$ are $\gamma$-Hölder, by Lemma 6.3 so is $D^{k-1} \log D \varphi_{i}$, with some Hölder constant $c_{0}$. Now we apply Lemma 6.4 to

$$
f_{n} \circ \cdots \circ f_{1}=\varphi_{y_{n}} \circ \cdots \circ \varphi_{y_{1}} \equiv \varphi_{n}^{y}
$$

and

$$
h_{j} \equiv D^{(k-1)} \log D \varphi_{y_{j}} .
$$

For $x \in I$, writing $x_{1}=x, x_{2}=h_{1}(x)$ etcetera as in Lemma 6.4 , since

$$
D^{(k-1)} \log D \varphi_{n}^{y}=\sum_{j=1}^{n} h_{j}\left(x_{j}\right),
$$

we conclude that $D^{(k-1)} \log D \varphi_{n}^{y}$ is $\gamma$-Hölder, with some different constant $c_{1}$ which is however independent of $n$. 
Now to prove the Theorem, first consider the case $k \geq 2$. Here we have $D^{(k-1)} \log D \Phi_{n}^{y}=D^{(k-1)} \log D \varphi_{n}^{y}$, since the constant derivative of $A_{n}^{y}$ disappears upon higher differentiation. For $k=1$ these are not equal; they differ by the constant $\log D A_{n}^{y}$ (which increases with $n$ ). However these cancel upon subtraction, so in either case we have, for any $a, b \in I$,

$$
\left|\log D \Phi_{n}^{y}(a)-\log D \Phi_{n}^{y}(b)\right|=\left|\log D \varphi_{n}^{y}(a)-\log D \varphi_{n}^{y}(b)\right| .
$$

Therefore for all $k \geq 1, D^{k-1} \log D \Phi_{n}^{y}$ is a sequence of bounded functions which is $\gamma$-Hölder with the same constant, $c_{1}$. Also, this sequence is uniformly bounded. For $k=1$ this follows from bounded distortion, as in the proof of Theorem 5.4, and in fact a bound is $e^{K \beta^{\gamma}}$. For $k>1$, we argue as follows: if it were unbounded, then by Hölder continuity with the same constant, some subsequence goes uniformly to either $+\infty$ or $-\infty$. By integration $(k-1)$ times, by induction this contradicts the boundedness for $k=1$. This implies equicontinuity. Now by boundedness and equicontinuity, there is some convergent subsequence, using the standard diagonalization argument as in the proof of the Arzela-Ascoli Theorem. At the same time, from $\S 5$ we know that $\lim D \Phi_{n}^{y}=D \Phi^{y}$ exists which implies $\log D \Phi_{n}^{y}$ converges to $\log D \Phi^{y}$. Calling the subsequence

$$
\log D \Phi_{n_{j}}^{y}=f_{j}
$$

we are in the situation of Lemma 6.5: $f_{j} \rightarrow f, D^{k-1} f_{j} \rightarrow g$ hence $D^{k-1} f=g$. Thus $D^{k-1} \log D \Phi^{y}$ is a uniform limit of $\gamma$-Hölder functions with the same constant $c_{o}$, hence the limit is $\gamma$-Hölder with constant $c_{o}$. From Lemma $6.3, D^{k} \Phi^{y}$ is also $\gamma$-Hölder and we are done for $k=1,2, \ldots$

Finally note that for $k=\infty$ we are done by part (i), and for $k=\omega$ we can apply Arzela-Ascoli to see that $\left\{\Phi_{n}^{y}\right\}$ is a normal family, hence the limit $\Phi^{y}$ is also analytic.

Remark: We emphasize again the subtle point in the logic of this argument: $\mathcal{C}^{1+\gamma}$ convergence of $\Phi_{n}^{y}$ to $\Phi^{y}$ is not known. What we $d o$ know is convergence in the $\mathcal{C}^{1}$ norm (from Theorem 5.9) and convergence along a subsequence in the $\mathcal{C}^{1+\gamma}$ norm, as just shown. This is enough to prove the claim of the Theorem.

\section{$\S 7$. Smoothness of limit sets, and rigidity.}

Given two $\mathcal{C}^{1+\gamma}$ hyperbolic Cantor sets $(C, S)$ and $(\widehat{C}, \widehat{S})$, recall that the (full) conjugacy $\Phi$ is an orderpreserving map defined on all of $I$. This map is uniquely determined on $C$ by the conjugacy equation, since, as one sees, the symbolic dynamics is preserved. Note that for any two topological Cantor sets, once they have been coded by the two-shift $\Sigma^{+}$in an order-preserving way, this conjugacy on the Cantor sets extends to a homeomorphism on $I$. The issue therefore is what types of conjugacies preserve what type of structure. As is well known and not hard to show, for instance, a biLipschitz $\Phi$ will preserve the Hausdorff dimension. We noted in [BF 1] that $\mathcal{C}^{1}$ maps preserve the order-two density. Furthermore for $\mathcal{C}^{1}$ conjugacy from [Su 1] one has:

Lemma 7.1. If two $\mathcal{C}^{1+\gamma}$ hyperbolic Cantor sets are $\mathcal{C}^{1}$ conjugate, then they have the same scaling function.

Proof. By uniform continuity of the derivatives, since we already know the scaling functions exist from Theorem 3.1, this is immediate.

Hence under the same assumption, by Corollary 5.3 we have:

Corollary 7.2. They have the same collection of limit sets.

To prove our rigidity theorem, we will need the following.

Lemma 7.3. Let $(C, S)$ be a hyperbolic $\mathcal{C}^{k+\gamma}$ Cantor set. Let $\widehat{S}: I_{0} \cup I_{1} \rightarrow I$ be a $\mathcal{C}^{k+\gamma}$ map such that $S=\widehat{S}$ on $C$. Then $S$ and $\widehat{S}$ are $\mathcal{C}^{k+\gamma}$ conjugate.

Proof. The conjugacy is the identity map on $C$; what we want to do is define it on the gaps. We begin by defining $\Phi$ to be the identity also on the gap $G$ between $I_{0}$ and $I_{1}$. The conjugacy is then uniquely defined from the conjugacy equation, by the dynamics. That is, writing $G_{x_{0} \ldots x_{n}}=\varphi_{x_{0} \ldots x_{n}}(G)$, we have for $a \in G_{x_{0} \ldots x_{n}}$,

$$
\Phi(a)=\widehat{\varphi}_{x_{0} \ldots x_{n}}\left(\varphi_{x_{0} \ldots x_{n}}^{-1}(a)\right)=\widehat{\varphi}_{x_{0} \ldots x_{n}}\left(S^{n}(a)\right) .
$$


One immediately checks that with this definition, $\Phi$ is a conjugacy.

This map is $\mathcal{C}^{k+\gamma}$ on the interiors of all the gaps. At points in $C$, to check $\mathcal{C}^{k+\gamma}$ one must be careful because these points are also limits of interior points in the gaps.

Here is one way of proving $\Phi$ is everywhere $\mathcal{C}^{k+\gamma}$.

Define a sequence of maps $\Phi_{n}: I \rightarrow I$ by: $\Phi_{0}=$ the identity, $\Phi_{1}=\Phi_{0}$ on $G$ and $\widehat{\varphi}_{x_{0}} \circ S$ everywhere else (i.e. on $I_{0} \cup I_{1}$ ), and inductively, set $\Phi_{n}$ to be equal to $\Phi_{n-1}$ everywhere except on $\bigcup I_{x_{0} \ldots x_{n}}$, where it is defined to be $\widehat{\varphi}_{x_{0} \ldots x_{n}} \circ S^{n}$.

These maps converge uniformly to $\Phi$. So if we can show that for each $n, D^{k} \Phi_{n}$ is $\gamma$-Hölder with a constant independent of $\mathrm{n}$, this will carry over to the limit and we will be done. (Here we will use the fact that the maps $\widehat{\varphi}_{x_{0} \ldots x_{n}} \circ S^{n}$ are $\gamma$-Hölder with a fixed constant).

The advantage of this method is that we must only check smoothness at each stage, and so each time at only finitely many points.

Now consider the map $f=\widehat{\varphi}_{x_{0} \ldots x_{n}} \circ S^{n}$ on $C \cap I_{x_{0} \ldots x_{n}}$. It is the identity there, and since $C$ is dense in itself, $D f=1$ on that set. Since it is twice differentiable, $D^{2} f=0$ there and similarly for $D^{k} f$. Therefore when we define $\Phi_{n}$ by gluing together $\widehat{\varphi}_{x_{0}, \ldots, x_{n-1}} \circ S^{n-1}$ and $\widehat{\varphi}_{x_{0} \ldots x_{n}} \circ S^{n}$ at an endpoint $p$, the two functions agree at $p$ for all derivatives $\leq k$. Also $D^{k}$ is $\gamma$-Hölder, for each piece. Hence for all $n, \Phi_{n}$ is $\mathcal{C}^{k+\gamma}$ with a fixed Hölder constant, as we wanted to show; so we are done.

This produces one conjugacy. In $\S 8$ we will return to this proof, in order to study how many such maps $\Phi$ there are.

We are now ready to prove:

Theorem 7.4 (highest smoothness). Given a $\mathcal{C}^{1+\gamma}$ hyperbolic Cantor set $C$, its limit sets have the highest degree of smoothness of any hyperbolic $\mathcal{C}^{1+\gamma}$ Cantor set in the $\mathcal{C}^{1+\gamma}$ - conjugacy class of $C$.

Proof. Let $(\widehat{C}, \widehat{S})$ be a $\mathcal{C}^{k+\gamma}, \mathcal{C}^{\infty}$ or $\mathcal{C}^{\omega}$ hyperbolic Cantor set which is $\mathcal{C}^{1}$ conjugate to $C$. By Corollary 7.2, $C$ and $\widehat{C}$ have the same limit sets. And by Theorem 6.1, the map $\widehat{\Phi}^{y}: I \rightarrow I$ defines a dynamics $S^{y}: I_{0}^{y} \cup I_{1}^{y} \rightarrow I$ by conjugation with the map $\widehat{S}$, which has that same degree of smoothness.

Theorem 7.5 (rigidity). If $(C, S)$ and $(\widehat{C}, \widehat{S})$ are two $\mathcal{C}^{k+\gamma}, \mathcal{C}^{\infty}$ or $\mathcal{C}^{\omega}$ hyperbolic Cantor sets which either (a) are $\mathcal{C}^{1}$ conjugate by a map $\Phi$, or (b) have the same scaling function $R$, then they are in fact conjugate by a map $\widetilde{\Phi}: I \rightarrow I$ which for (a) agrees with $\Phi$ on $C$ or for (b) agrees with the coding; this map is $\mathcal{C}^{k+\gamma}$, $\mathcal{C}^{\infty}$ or $\mathcal{C}^{\omega}$ respectively.

Proof. By either hypothesis they have the same limit sets. Choose one, $C^{y}$. Again by Theorem 6.1, the maps $\Phi^{y}, \widehat{\Phi}^{y}$ have the same smoothness as $S, \widehat{S}$. Now let $S^{y}, \widehat{S}^{y}$ denote the maps defined on $I_{0}^{y} \cup I_{1}^{y}$ by these conjugacies. We are exactly in the situation of Lemma 7.3, and have a conjugacy $\Phi$ of $S^{y}$ and $\widehat{S}^{y}$. Composing the three maps

$$
\left(\widehat{\Phi}^{y}\right)^{-1} \circ \Phi \circ \Phi^{y}
$$

finishes the proof.

\section{$\S 8$ Banach space structure.}

Fix a hyperbolic $\mathcal{C}^{1+\gamma}$ Cantor set $(C, S)$. For $r=k+\gamma$ where $\gamma \in(0,1], k \geq 1$ or for $r=\infty$, $\omega$ we write $\mathcal{E}^{r} \equiv \mathcal{E}^{r}(C)$ for the collection of Cantor sets (with maps) which are $\mathcal{C}^{r}$ - conjugate to $(C, S)$. (From Lemma 1.1.2, these are also hyperbolic $\mathcal{C}^{1+\gamma}$ Cantor sets). The spaces Diff ${ }^{r}, \widehat{\mathcal{E}}^{r}, \mathcal{E}_{*}^{r}$ and $\mathcal{E}_{* *}^{r}$ are defined as they were in $\S 4$ for the case $r=1+\gamma$.

In this section we will see how $\widehat{\mathcal{E}}^{r}$ can be viewed as a Banach manifold, in fact a Banach Lie group. We will also define a natural topology on $\mathcal{E}^{r}$, and show that $\widehat{\mathcal{E}}^{r}$ factors nicely over $\mathcal{E}^{r}$ as a topological space.

We define first the $\mathcal{C}^{\gamma}$ norm on the Hölder functions $\mathcal{C}^{\gamma}(I, \mathbb{R})$ to be

$$
\|f\|_{\mathcal{C} \gamma}=\|f\|_{\infty}+\sup _{x, y \in I} \frac{|f(x)-f(y)|}{|x-y|^{\gamma}} .
$$

For $r=k+\gamma$ where $\gamma \in(0,1], k \geq 1$, the $\mathcal{C}^{r}$ norm will be

$$
\Sigma_{l=0}^{k-1}\left\|D^{l} f\right\|_{\infty}+\left\|D^{k} f\right\|_{\mathcal{C} \gamma} .
$$


For $\mathcal{C}^{\infty}$ we define

$$
\|f\|_{\mathcal{C} \infty}=\sup _{l}\left\{\left\|D^{l} f\right\|_{\infty}\right\}
$$

and for $\mathcal{C}^{\omega}$ we will use the sup norm (since it is equivalent to all the other $\mathcal{C}^{r}$ norms there).

By definition a Banach manifold is a manifold which is locally modelled on a Banach space, and a Lie group is a group, which is also a $C^{\infty}$ manifold modelled on a complete, locally convex vector space (see e.g. [Mi]). Recall that Diffr denotes the $\mathcal{C}^{r}$ order-preserving diffeomorphisms of $I$. Now choice of a set in $\mathcal{E}^{r}$ identifies the collection $\widehat{\mathcal{E}}^{r}$ with Diff ${ }^{r}$, as we have seen in $\S 4$. Diffr is an open subset of $\mathcal{C}_{0,1}^{r}(I, \mathbb{R})$, which is how we will write the set of all $\mathcal{C}^{r}$ functions from $I$ to $\mathbb{R}$ such that $f(0)=0$ and $f(1)=1$. This in turn is a closed affine subspace of $\mathcal{C}^{r}(I, \mathbb{R})$. To see this note that, defining

$$
\mathcal{B}_{0,1}^{r}(I, \mathbb{R})=\left\{f \in \mathcal{C}^{r}(I, \mathbb{R}): f(0)=0=f(1)\right\},
$$

two functions in $\mathcal{C}_{0,1}^{r}$ differ exactly by an element of $\mathcal{B}_{0,1}^{r}$. Now $\mathcal{B}_{0,1}^{r}$ is a Banach space, with the $\mathcal{C}^{r}$ norm. Hence $\widehat{\mathcal{E}}^{r}$ is a Banach manifold: it is identified with Diff ${ }^{r}$, which in turn corresponds to an open subset of $\mathcal{B}_{0,1}^{r}$. Now Diff $r$ is a group, hence it (and therefore $\widehat{\mathcal{E}}^{r}$ ) is a Banach Lie group. Two choices have been made: the choice of a Cantor set in $\mathcal{E}^{r}$, and of a special point (the identity) in Diff ${ }^{r}$. These choices determined the maps to $\mathcal{B}_{0,1}^{r}$ and hence the metric (inherited from the $\mathcal{C}^{r}$ norm). Both choices moreover amount to the same thing: changing $C$ to $D$ in $\mathcal{E}^{r}$ (as in Proposition 5.1, for $r=1$ ) corresponds to a right translation in the group Diffr .

Now in a Lie group one ideally would like to work with a (left- or right-) invariant metric. If the group is compact (or, more generally, amenable), one can make a given metric invariant (while keeping equivalence) by averaging over translations. In our case, however, one cannot get an equivalent invariant metric- Diff ${ }^{r}$ is not only non-compact but non-amenable! The (non-uniform) bounded equivalence proved in Proposition 5.1 is nevertheless enough for what we needed, for the proof of Theorem 5.3.

In summary we have:

Proposition 8.1. $\widehat{\mathcal{E}}^{r}$ is a Banach manifold. It is naturally identified up to right composition with the Banach Lie group Diffr ${ }^{r}$, and with an open subset of a closed affine subspace of $\mathcal{C}^{r}(I, \mathbb{R})$.

A similar estimate to that shown in Proposition 5.1 for $r=1$ holds for $r>1$. Therefore one has, for $r=k+\gamma, \infty, \omega:$

Proposition 8.2.

(a) The $\mathcal{C}^{r}$ metric on Diff ${ }^{r}$ is right-invariant up to (non-uniform) bounded equivalence.

(b) The $\mathcal{C}^{r}$ metric on $\widehat{\mathcal{E}}^{r}$ is base-point independent up to (non-uniform) bounded equivalence.

We note that $\mathcal{E}_{*}^{r}$, the space of marked Cantor sets, is also a Banach manifold, by the same reasoning as for Diffr : it is an open subset of a closed affine subspace of $\mathcal{C}^{r}(C, \mathbb{R})$.

Next we will describe more fully the relationship between the spaces $\widehat{\mathcal{E}}^{r}$ and $\mathcal{E}^{r}$. For $r=k+\gamma$, we write $\operatorname{Diff}_{0}^{r}(I)$ for the collection of $\mathcal{C}^{r}$ diffeomorphisms of the unit interval $I$ whose first $k$ derivatives are $1,0, \ldots, 0$ at the endpoints. This is also a Banach manifold.

Proposition 8.3. Given the choice of a Cantor set $C, \widehat{\mathcal{E}}^{r}$ factors naturally, set theoretically and topologically, as

$$
\widehat{\mathcal{E}}^{r}=\mathcal{E}^{r} \times \operatorname{Diff}_{0}^{r}(I)
$$

with the topology on $\mathcal{E}^{r}$ defined below.

Proof. First, let us consider how many $\mathcal{C}^{r}$ maps from $I$ to $I$ there are, conjugating $(C, S)$ with $(C, \widehat{S})$ (the Cantor sets are the same, but the maps may be different off of the Cantor sets). In the proof of Lemma 7.3 , note that instead of starting with $\Phi$ equal to the identity on the gap $G$ we could have taken any $\mathcal{C}^{k+\gamma}$ diffeomorphism from $G$ to itself, whose derivatives agree with the identity at the endpoints, up to order $k$. Conversely any conjugacy is specified by its values on $G$, since elsewhere it is then determined by the 
dynamics. Therefore we see that the set of $\mathcal{C}^{r}$ conjugacies from $(C, S)$ to $(C, \widehat{S})$ correspond naturally to $\operatorname{Diff}_{0}^{r}(I)$. (This is also true when $S=\widehat{S} !$ )

Next we consider how many different $\mathcal{C}^{r}$ conjugacies are possible in the rigidity theorem, from $(C, S)$ to $(\widehat{C}, \widehat{S})$. Given the existence of one such map and hence a restricted conjugacy, we can define (all the) other extensions by a method like that used in the proof just given. That is, we first define the conjugacy arbitrarily on the first-level gaps (but with the correct derivatives of order $\leq k$ at the endpoints). Then we extend by the dynamics. Or, we can quote that statement directly, making use of use a ratio Cantor set as intermediary as in the proof of Theorem 7.5, and now replacing $\Phi$ by one of the more general maps described above.

This shows we have a product of sets. $\mathcal{E}^{r}$ has not yet been given a topology. But from the product decomposition, we can define a family of metrics as follows. Choosing one element of Diffr defines an embedding into $\widehat{\mathcal{E}}^{r}$, and we just use the $\mathcal{C}^{r}$ metric there. (One would like to get a more natural definition by taking the infimum over all such choices; however, it is then not clear that the triangle inequality will hold). At any rate the metrics are equivalent, so this defines a natural topology on $\mathcal{E}^{r}$.

We will show that the $\mathcal{C}^{r}$ metric on $\widehat{\mathcal{E}}^{r}$ is equivalent to the product of the metrics on $\mathcal{E}^{r}$ and Diffr.

It is easy to see that the map from $\mathcal{E}^{r}$ to each factor is continuous. (To Diffr it is also affine). For the converse, given the base point $(C, S)$, let first $f, g \in \operatorname{Diff}^{r}$ be such that $\left(C_{f}, S_{f}\right)=\left(C_{g}, S_{g}\right)$. Write $f_{0}, g_{0}$ for the corresponding elements of Diffr ${ }_{0}^{r}$, i.e. the restrictions of $f$ and $g$ to the middle gap of $C$ (rescaled in the range). We claim that if $f_{0}$ and $g_{0}$ are close in Diffr then $f$ and $g$ are close in Diff ${ }^{r}$. The formula for $f$ on an $n^{\text {th }}$ level gap of $C$ is

$$
f(a)=\varphi_{x_{0} \ldots x_{n}}^{f} \circ f_{0} \circ \varphi_{x_{0} \ldots x_{n}}^{-1}(a) .
$$

Here $\varphi, \varphi^{f}$ denote inverse branches for $S$ and $S_{f}$ respectively. By assumption $\varphi^{f}=\varphi^{g}$. Now by bounded distortion (Lemma 6.4) for $k=1$, and for general $k$ by the proof of Theorem $6.1, D^{k} \varphi_{x_{0} \ldots x_{n}}^{f}$ is uniformly $\gamma-$ Hölder with constant independent of $n$. This proves $\|f-g\|_{\mathcal{C} 1}$ is small, which is what we wanted to show.

Next we drop the assumption that $f$ and $g$ give the same maps. We have chosen an element of Diff ${ }_{0}^{r}$ to define the metric on $\mathcal{E}^{r}$. Let $\tilde{f}, \tilde{g}$ denote the maps in Diff ${ }^{r}$ such that $\tilde{f}_{0}=\tilde{g}_{0}$ is that element, with $\left(C_{f}, S_{f}\right)=\left(C_{\tilde{f}}, S_{\tilde{f}}\right)$ and similarly for $g$. Now by definition, the distance between the pairs in $\mathcal{E}^{r}$ is $\|\tilde{f}-\tilde{g}\|_{\mathcal{C} 1}$. So we just apply the triangle inequality using the previous case, to conclude that $d_{C}\left(C_{f}, C_{g}\right) \equiv\|f-g\|_{\mathcal{C} 1}$ is also small.

Remark. In a conversation about the proof of Lemma 7.3, Yair Minsky pointed out to us an interesting parallel between that argument and Sullivan's "flexibility and rigidity" theorem for Kleinian groups. Sullivan showed, for a finitely generated Kleinian group $\Gamma$, that the limit set $\Lambda$ of the group itself is "rigid", i.e. a quasiconformal conjugacy (to another Kleinian group) which lives (Lebesgue almost-surely) on $\Lambda$ must be Möbius. This is a consequence of Sullivan's lemma that $\Lambda$ carries no measurable $\Gamma$ - invariant line fields. (Note that by contrast, for hyperbolic Cantor sets, quasisymmetric conjugacy does not imply smooth conjugacy; as we have seen, one also needs to know the scaling function).

Sullivan used this to show that a quasiconformal conjugacy is determined by a Beltrami differential on $\Omega / \Gamma$ where $\Omega$ is the domain of discontinuity. Thus, in a sense, one has rigidity on the limit set and flexibility off of it. The group actions correspond to the two (not restricted) expanding maps, and the surface $\Omega / \Gamma$, or equivalently a fundamental domain for the action of $\Gamma$ on $\Omega$, is analogous to the gap $G$ of the Cantor set. As is the case there, the conjugacy is then specified elsewhere by the dynamics. Sullivan's theorem can then be stated as follows: $\operatorname{Teich}(\Gamma)=\operatorname{Teich}(\Omega / \Gamma)$, where this refers to the Teichmüller space of a group and of a surface respectively; this formulation led to the statement in the Proposition above.

Concluding remarks: limit sets as the attractor of a semigroup action. $\mathcal{E}^{1+\gamma}$ denotes the $\mathcal{C}^{1+\gamma}$ equivalence class of a given $\mathcal{C}^{1+\gamma}$ hyperbolic Cantor set. The nested subclasses $\mathcal{E}^{r}$, for maps of smoothness $r=k+\gamma, \infty, \omega$, also are conjugate with that higher degree of smoothness. Thus smoothness classes are also conjugacy classes. Choosing one set in $\mathcal{E}^{r}$ as a base point, $\mathcal{E}^{r}$ is naturally identified with a topological factor of the $\mathcal{C}^{r}$ orientation-preserving diffeomorphisms of the interval, Diff ${ }^{r}$ which is a Banach manifold. Moreover we can choose one Cantor set as a common base point for all the $\mathcal{E}^{r}$, since by Theorem 7.4 smoothest Cantor sets exist. Then the nested collections $\mathcal{E}^{1+\gamma} \supseteq \cdots \supseteq \mathcal{E}^{r} \ldots$ are naturally identified with factors of 
Diff $^{1+\gamma} \supseteq \cdots \supseteq \operatorname{Diff}^{r} \ldots$ (Each is a Banach manifold with its own topology, and is a dense subset of the larger collections, with respect to their topologies). The spaces of marked Cantor sets $\mathcal{E}_{*}^{r}$ are also Banach manifolds. The free semigroup on two generators $F S_{2}$ acts on each submanifold $\mathcal{E}_{*}^{r}$ by replacing it with its left or right Cantor subset. From Theorem 7.4, the limit sets are in the intersection of the $\mathcal{E}_{*}^{r}$. From Theorem 5.3, because the bounds are uniform over all Cantor subsets of level $n$, the collection of limit sets is an attractor for this action. This convergence is exponentially fast in the $\mathcal{C}^{1}$ norm. (Warning: we have only shown convergence in this norm; see the Remark at the end of $\S 6$ ). The semigroup action on the attractor itself can be described symbolically very simply as follows. Recall the map $y \mapsto C^{y}$ for $y$ in the dual Cantor set $\Sigma^{-}$and $C^{y}$ the corresponding ratio Cantor set. Now just concatenate $y$ on the right with a finite string of symbols.

\section{REFERENCES}

[AF] P. Arnoux and A.M. Fisher, Renormalization, scenery and the Teichmüller mapping flow (in preparation).

[Be] T. Bedford, Applications of dynamical systems theory to fractal sets: a study of cookie cutter sets, Proceedings of the Séminaire de mathématiques supérieures "Fractal Geometry and analysis", Université de Montréal, NATO ASI Series, Kluwer, Amsterdam.

[BF 1] T. Bedford and A.M. Fisher, Analogues of the Lebesgue density theorem for fractal sets of reals and integers, Proc. London Math. Soc. 64 (1992), 95-124.

[BF 2] T. Bedford and A.M. Fisher, On the magnification of Cantor sets and their limit models (to appear), Monatsh. Math.

[BF 3] T. Bedford and A.M. Fisher, Models for the scenery flow of a hyperbolic Cantor set (in preparation).

[BFU] T. Bedford, A.M. Fisher and M. Urbanski, The scenery flow for hyperbolic Julia sets (in preparation).

[Bo 1] R. Bowen, Equilibrium states and the ergodic theory of Anosov diffeomorphisms, Lecture Notes in Mathematics 470, Springer Verlag, 1975.

[Bo 2] R. Bowen, Hausdorff dimension of quasi-circles, Publications Mathématiques (Institut des Hautes Etudes Scientifiques, Paris) 50 (1979), 11-25.

[Bu-F] M Burger and A.M. Fisher, Infinite measure unique ergodicity for the horocycle flows of certain Fuchsian groups (in preparation).

[Fi 1] A.M. Fisher, Integer Cantor sets and an order-two ergodic theorem, Ergod. Th. and Dynam. Sys. 13 (1992), 45-64.

[Fi 2] A.M. Fisher, Analogues of geodesic and horocycle flows for certain fractal sets (in preparation).

[Fu] H. Furstenberg, Intersections of Cantor sets and transversality of semigroups, Problems in Analysis, a symposium in honor of S. Bochner, Princeton University Press, pp. 41-59.

[deM-vS] W. de Melo and S. J. van Strien, One-Dimensional Dynamics, Springer-Verlag, 1993.

[Mand] B. Mandelbrot, The fractal geometry of nature, W.H. Freeman, 1983.

[Mané] R. Mañé, Ergodic theory and differentiable systems, Springer Verlag, 1987.

[Mi] J. Milnor, Remarks on infinite-dimensional Lie groups, Relativity, groups and topology II, Elsevier, 1984.

[P] A. Pinto, Convergence of Renormalisation and Rigidity of Dynamical Systems (PhD Thesis, Warwick University), 1991.

[PR 1] A. Pinto and D. A. Rand, A characterisation of the moduli space of smooth Markov maps by horizontal scaling functions (preprint).

[PR 2] A. Pinto and D. A. Rand, A classification of $\mathcal{C}^{1+\alpha}$ structures on embedded trees (preprint).

[Ra] D. A. Rand, Global phase space universality, smooth conjugacies and renormalization: I. The $\mathcal{C}^{1+\alpha}$ case, Nonlinearity 1 (1988), 181-202.

[Ru] D. Ruelle, Repellers for real analytic maps, Ergod. Th. and Dynam. Sys. 2 (1982), 99-107.

[Sh-Su] M. Shub and D. Sullivan, Expanding endomorphisms of the circle revisited, Ergod. Th. and Dynam. Sys. 5 (1985), 285-289.

[Su 1] D. Sullivan, Differentiable structures on fractal-like sets, determined by intrinsic scaling functions on dual Cantor sets, AMS Proc. Symp. Pure Math. 48 (1987), 15-23.

[Su 2] D. Sullivan, Bounds, quadratic differentials, and renormalization conjectures, AMS Centennial Publications 2 (1991).

[Su 3] D. Sullivan, Linking the universalities of Milnor-Thurston Feigenbaum and Ahlfors-Bers, Topological Methods in Modern Mathematics, Publish or Perish, 1992, pp. 543-564.

[T] Tan Lei, Similarity between the Mandelbrot set and Julia sets, Comm. Math. Phys. 134 (1990), 587-617.

[TP] F. Tangerman and F. Przytycki, Cantor sets on the line: scaling functions and smoothness of the shift map (preprint, Stony Brook IMS no. 1992/6). 